\title{
Exact Dynamics of Nonadditive Environments in Non-Markovian Open Quantum Systems
}

\author{
Dominic Gribben $\odot,{ }^{1, \dagger}$ Dominic M. Rouse $\odot,{ }^{1, \dagger}$ Jake Iles-Smith $\odot,{ }^{2,3, \dagger}$ Aidan Strathearn, ${ }^{4}$ \\ Henry Maguire, ${ }^{2}$ Peter Kirton $\odot,{ }^{5}$ Ahsan Nazir $\odot,{ }^{2}$ Erik M. Gauger $\odot,{ }^{6}$ and Brendon W. Lovett $\odot{ }^{1, *}$ \\ ${ }^{1}$ SUPA, School of Physics and Astronomy, University of St Andrews, St Andrews KY16 9SS, United Kingdom \\ ${ }^{2}$ Department of Physics and Astronomy, The University of Manchester, Oxford Road, Manchester M13 9PL, \\ United Kingdom \\ ${ }^{3}$ Department of Electrical and Electronic Engineering, The University of Manchester, Sackville Street Building, \\ Manchester M1 3BB, United Kingdom \\ ${ }^{4}$ School of Mathematics and Physics, The University of Queensland, St Lucia, Queensland 4072, Australia \\ ${ }^{5}$ Department of Physics and SUPA, University of Strathclyde, Glasgow G4 ONG, United Kingdom \\ ${ }^{6}$ SUPA, Institute of Photonics and Quantum Sciences, Heriot-Watt University, Edinburgh EH14 4AS, United \\ Kingdom
}

(Received 17 September 2021; revised 21 December 2021; accepted 7 January 2022; published 7 February 2022)

When a quantum system couples strongly to multiple baths, then it is generally no longer possible to describe the resulting system dynamics by simply adding the individual effects of each bath. However, capturing such multibath system dynamics typically requires approximations that can obscure some of the nonadditive effects. Here we present a numerically exact and efficient technique for tackling this problem that builds on the time-evolving matrix product operator (TEMPO) representation. We test the method by applying it to a simple model system that exhibits nonadditive behavior: a two-level dipole coupled to both a vibrational and an optical bath. Although not directly coupled, there is an effective interaction between the baths mediated by the system that can lead to population inversion in the matter system when the vibrational coupling is strong. We benchmark and validate multibath TEMPO against two approximate methods - one based on a polaron transformation, the other on an identification of a reaction coordinate - before exploring the regime of simultaneously strong vibrational and optical coupling where the approximate techniques break down. Here we uncover a new regime where the quantum Zeno effect leads to a fully mixed state of the electronic system.

DOI: 10.1103/PRXQuantum.3.010321

\section{INTRODUCTION}

Open quantum systems are often significantly coupled to more than one kind of environment. However, the combined influence of these different environments is generally more than the sum of their individual parts: it is thus crucial to account for nonadditive effects - that is, effects that originate from an interplay between two or more competing environments [1-5].

Examples of nonadditive behavior include those seen or predicted in optically active quantum systems that

\footnotetext{
*bw14@st-andrews.ac.uk

†These authors contributed equally.
}

Published by the American Physical Society under the terms of the Creative Commons Attribution 4.0 International license. Further distribution of this work must maintain attribution to the author(s) and the published article's title, journal citation, and DOI. are strongly influenced by their vibrational environments, which are ubiquitous in condensed matter and molecular physics. Not only do vibrational interactions lead to complex dynamical behavior, they are central in determining the optical and electronic properties of a system. For example, they are thought to play a key role in light harvesting and energy transfer [6-10]. Another example occurs in molecular nanojunctions, where the combined effect of the leads and vibrational environments is nonadditive [11,12], and using an additive treatment can even lead to a violation of the Carnot bound on efficiency [13]. Using additive treatments can lead to other unphysical predictions too, for example anomalous emission of photons from the ground state in regimes of strong light-matter coupling [14]. Furthermore, it can miss key dynamical and steady-state behavior, such as the Franck-Condon blockade observed in quantum transport $[15,16]$.

However, typical standard perturbative methods such as Redfield theory do make an implicit additive 
approximation, such that the impact of each individual environment is mutually independent. Such a treatment will fail in general once the magnitude of the system-bath interaction Hamiltonians for each environment becomes strong, when non-Markovian effects are important. A number of techniques have been developed to capture nonadditive and strong coupling effects, while maintaining the conceptual simplicity of Born-Markov master equations, such as the polaron transformation [17-22] and its extensions [23-25]; methods based on the pseudomode approach [26-28] and its generalization [29-31]; and the reaction coordinate $(\mathrm{RC})$ mapping $[4,32,33]$. Even though these methods can access nonperturbative regimes, they are typically limited to a particular parameter regime due to the approximations required in their derivation.

To overcome these kinds of limitations, tensor network techniques have become increasingly useful in reducing the computational cost and time of numerically exact calculations of open quantum system dynamics [34]. Although originally formulated to more efficiently represent many-body states with area law entanglement $[35,36]$ they are of more general use in problems that can be cast in a form where the compression of large tensors gives a significant numerical advantage. For example, chain-mapping techniques [37,38] involve transforming the topology of system-bath couplings from a star to a one-dimensional (1D) chain with nearest-neighbor couplings; this can then be efficiently represented as a spatial matrix product state (MPS), which also works at finite bath temperature [39]. An alternative approach is to construct a MPS in time able to efficiently capture non-Markovian correlations in an equivalent fashion that spatial MPS capture spatial correlations $[40,41]$. The resulting time-evolving matrix product operator (TEMPO) method provides an efficient approach for calculating the exact dynamics of an open quantum system interacting with a complex environment.

In this work we describe how to formulate multibath TEMPO, and so describe a numerically exact approach for capturing the effects of strongly coupling environments that are nonadditive. As an example calculation using this new approach, we investigate the nonequilibrium behavior of a two-level quantum emitter interacting with both vibrational and optical environments. We find that multibath TEMPO allows us to explore parameter regimes that are difficult to access with standard approximate techniques. We first confirm the behavior predicted in Ref. [4], where nonadditive effects lead to a population inversion if the optical temperature and vibrational coupling strength are large enough. We then use multibath TEMPO to access the difficult regime of simultaneously strong optical and vibrational coupling. Here we find that the steady-state population of both states of the emitter is fixed at one half regardless of temperature; we associate this with a quantum Zeno effect arising from the strong optical coupling. This serves to not only prove the importance of nonadditive effects in the behavior of open quantum systems, but also reveals new unexpected behavior in the nonequilibrium steady state of the system. By comparing TEMPO to the polaron and RC theories, we gain analytic insight into the otherwise purely numerical approach, as well as determine when such approximate techniques are accurate.

In the next section we describe the multibath TEMPO technique in detail. In Sec. III we set out the example calculation, introducing the model of a single dipole coupled to both an optical and vibrational bath. We also show, in a simplified description, the mechanism by which population inversion can occur. In Sec. IV, we summarize the polaron transformation and RC mapping techniques, which we compare to the exact results obtained from multiplebath TEMPO in Sec. V. We explore when the approximate techniques are accurate, and present results that can only be captured using our new approach. Our conclusions are drawn in Sec. VI.

\section{MULTIBATH TEMPO}

In this section we present a derivation of the TEMPO algorithm extended to simulate a system coupled to multiple baths with noncommuting operators. A key step in the original path integral framework $[40,42,43]$ is to insert the complete eigenbasis of the coupling operator between each timestep of a discretized influence functional. A different approach must be taken in the general case of multiple coupling operators that do not share an eigenbasis, and this is discussed in Ref. [44]. However, a viable method of contracting the resulting influence tensor is still required, and we provide this here. In particular, we enable efficient contraction of this influence tensor by decomposing it into contributions from each environment, allowing us to construct the influence tensor for each independently. This is distinct from the independent calculation, and subsequent addition, of dynamical generators. In combining the separate influence tensors the total, nonadditive effect of the environments is faithfully recovered provided this is done on a fine enough timescale. In Fig. 1 we show this process schematically for the specific model we will consider from Sec. III A of this paper: a two-level system coupled to two environments. This process is made tractable by employing efficient tensor network representations $[35,36]$ of both the separate environmental contributions and the combined effect of all these environments and the internal system Hamiltonian $[41,45]$.

\section{A. Multibath influence functional}

The generic Hamiltonian we consider is

$$
H=H_{S}+\sum_{\alpha}\left(H_{I \alpha}+H_{B \alpha}\right)
$$


(a)

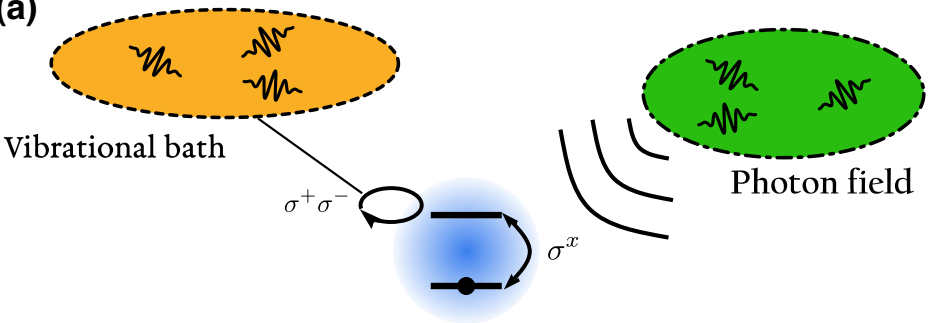

(b)

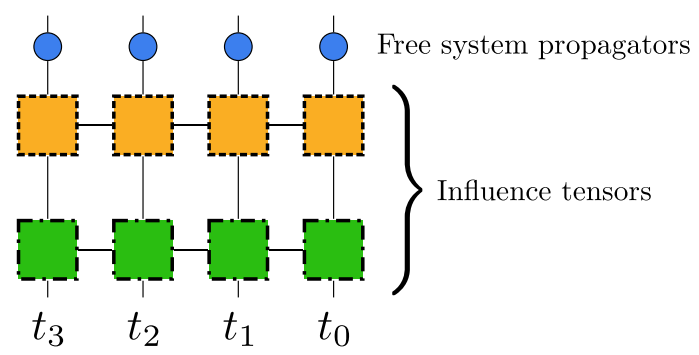

FIG. 1. (a) Sketch of the model considered in this paper: a two-level dimer coupled to both a vibrational bath and a photon field. (b) Schematic tensor network representation of our technique for nonperturbative simulation of the dynamics. By considering how the system evolves timestep by timestep, there is a contribution from both the time-local free system propagators and the influence tensors for each environment that account for any non-Markovian effects induced by the interactions.

where $H_{S}$ is the (arbitrary) system Hamiltonian, and

$$
H_{I \alpha}=s_{\alpha} \sum_{q}\left(g_{\alpha q} a_{\alpha q}+g_{\alpha q}^{*} a_{\alpha q}^{\dagger}\right)
$$

and

$$
H_{B \alpha}=\sum_{q} \omega_{\alpha q} a_{\alpha q}^{\dagger} a_{\alpha q}
$$

are the interaction and free bath Hamiltonians, respectively. Here $a_{\alpha q}\left(a_{\alpha q}^{\dagger}\right)$ is the annihilation (creation) operator corresponding to mode $q$ in bath $\alpha$ with frequency $\omega_{\alpha q}$. The arbitrary system operator $s_{\alpha}$ couples to mode $q$ in bath $\alpha$ with strength $g_{\alpha q}$. The baths can be continuous or discrete; in this paper we consider two continua. We assume that the initial state is separable into system and bath terms with the baths each initially in a Gaussian state, e.g., thermal equilibrium at temperatures $T_{\alpha}$. Throughout this paper we will use $\hbar=k_{B}=1$.

We work in a representation where the $d \times d$ density matrix $\rho$, an operator on the Hilbert space $\mathcal{H}$ spanned by basis vectors $\left\{\left|e_{i}\right\rangle\right\}$, is mapped to a $d^{2}$ vector in Liouville space $\mathfrak{L}=\mathcal{H} \otimes \mathcal{H}$ spanned by $\left\{\left|e_{i}\right\rangle \otimes\left|e_{j}\right\rangle\right.$. Formally, this mapping corresponds to

$$
\begin{gathered}
\left.\rho=\sum_{i j} \rho_{i j}\left|e_{i}\right\rangle\left\langle e_{j}|\rightarrow| \rho\right\rangle\right\rangle=\sum_{i j} \rho_{i j}\left|e_{i}\right\rangle \otimes\left|e_{j}\right\rangle, \\
\left.\left.O \rho \rightarrow O^{L}|\rho\rangle\right\rangle=O \otimes \mathbb{I}|\rho\rangle\right\rangle, \\
\left.\left.\rho O \rightarrow O^{R}|\rho\rangle\right\rangle=\mathbb{I} \otimes O^{T}|\rho\rangle\right\rangle .
\end{gathered}
$$

For convenience, we proceed in the interaction picture with respect to $H_{S}$ and $H_{B \alpha}$. The von Neumann equation is then

$$
\left.\left.\frac{d}{d t}|\rho(t)\rangle\right\rangle=\sum_{\alpha} \mathcal{L}_{I \alpha}(t)|\rho(t)\rangle\right\rangle,
$$

where we have introduced the Liouvillian superoperators $\mathcal{L}_{I \alpha}=-i\left(H_{I \alpha}^{L}-H_{I \alpha}^{R}\right)$. The exact solution of the von Neumann equation can be written as

$$
\begin{aligned}
|\rho(t)\rangle\rangle & \left.=\overleftarrow{T} \exp \left(\int_{0}^{t} \sum_{\alpha} \mathcal{L}_{I \alpha}\left(t^{\prime}\right) d t^{\prime}\right)|\rho(0)\rangle\right\rangle \\
& =\Lambda(t)|\rho(0)\rangle\rangle
\end{aligned}
$$

where $\overleftarrow{T}$ signifies that superoperators be time ordered from right to left and $\Lambda(t)$ is the propagator. We now factorize the propagator into a product of exponentials for each bath,

$$
\Lambda(t)=\overleftarrow{T} \prod_{\alpha} \exp \left(\int_{0}^{t} \mathcal{L}_{I \alpha}\left(t^{\prime}\right) d t^{\prime}\right)
$$

which is possible because integrals of operators under time ordering commute.

We define $\langle\cdot\rangle_{\alpha}$ as the expectation taken with respect to the initial state of bath $\alpha$ and set $\left\langle\mathcal{L}_{I \alpha}\right\rangle_{\alpha}=0$ for all $\alpha$ without loss of generality. Each bath can now be traced out independently using $\langle\exp (X)\rangle=\exp \left(\left\langle X^{2}\right\rangle / 2\right)$, true for any variable $X$ with a Gaussian distribution and zero mean. With this, and using idempotency of time ordering $\overleftarrow{T}=$ $\overleftarrow{T} \overleftarrow{T}$, we trace out the baths in Eq. (9) to leave a dynamical map for the reduced system alone:

$$
\begin{aligned}
\Lambda_{S}(t) & =\overleftarrow{T} \prod_{\alpha} \exp \left(\int_{0}^{t} \int_{0}^{t^{\prime}}\left\langle\mathcal{L}_{I \alpha}\left(t^{\prime}\right) \mathcal{L}_{I \alpha}\left(t^{\prime \prime}\right)\right\rangle_{\alpha} d t^{\prime} d t^{\prime \prime}\right) \\
& =\overleftarrow{T} \prod_{\alpha} \mathcal{F}_{\alpha}\left[s_{\alpha}^{L}, s_{\alpha}^{R}\right]
\end{aligned}
$$

Here we have enacted the time ordering of the bath operators within the exponents in the first line, gaining a factor of 2. We have also defined the system superoperatorvalued influence functionals, $\mathcal{F}_{\alpha}\left[s_{\alpha}^{L}, s_{\alpha}^{R}\right]$, where $s_{\alpha}^{L}$ and $s_{\alpha}^{R}$ 
are the left and right acting system superoperators corresponding to operator $s_{\alpha}$, as defined in Eqs. (5) and (6).

The system superoperator-valued expectations of the interaction Liouvillians in Eq. (10) are readily evaluated. We obtain

$$
\begin{aligned}
\left\langle\mathcal{L}_{I \alpha}\left(t^{\prime}\right) \mathcal{L}_{I \alpha}\left(t^{\prime \prime}\right)\right\rangle_{\alpha}= & -s_{\alpha}^{-}\left(t^{\prime}\right)\left[s_{\alpha}^{-}\left(t^{\prime \prime}\right) C_{R}^{\alpha}\left(t^{\prime}-t^{\prime \prime}\right)\right. \\
& \left.-i s_{\alpha}^{+}\left(t^{\prime \prime}\right) C_{I}^{\alpha}\left(t^{\prime}-t^{\prime \prime}\right)\right],
\end{aligned}
$$

where $s_{\alpha}^{ \pm}=s_{\alpha}^{L} \pm s_{\alpha}^{R}$, and $C_{R}^{\alpha}(t)$ and $C_{I}^{\alpha}(t)$ are the real and imaginary parts of

$$
C^{\alpha}(t)=\sum_{q}\left|g_{\alpha q}\right|^{2}\left[\cos \left(\omega_{\alpha q} t\right) \operatorname{coth}\left(\frac{\omega_{\alpha q}}{2 T_{\alpha}}\right)-i \sin \left(\omega_{\alpha q} t\right)\right],
$$

the autocorrelation function of bath $\alpha$ [46].

\section{B. Time ordering using tensor contractions}

Here we present the formalism required to cast Eq. (10) into a tensor network, closely following that introduced in Ref. [44]. To simplify our presentation, we combine the influence functionals for each bath in Eq. (10) into a single multibath influence function. Now we discretize the double integrals appearing in this influence functional onto a grid of timesteps, $t_{k}=k \Delta$, where $0 \geq k \geq N$. This allows us to write

$$
\Lambda_{S}\left(t_{N}\right) \approx \overleftarrow{T} \prod_{k=0}^{N} \prod_{k^{\prime}=0}^{k} \mathcal{I}_{k-k^{\prime}}\left(t_{k}, t_{k^{\prime}}\right)
$$

where

$$
\mathcal{I}_{k-k^{\prime}}\left(t_{k}, t_{k^{\prime}}\right)=\exp \left(\sum_{\alpha}\left\langle\mathcal{L}_{I \alpha}\left(t_{k}\right) \mathcal{L}_{I \alpha}\left(t_{k^{\prime}}\right)\right\rangle_{\alpha} \Delta^{2}\right)
$$

is a system superoperator-valued quantity that determines the influence of timestep $t_{k^{\prime}}$ on $t_{k}$. Operators nonlocal in space generate spatial correlations when acting on a state and here we can equivalently think of the time nonlocal superoperators $\mathcal{I}_{k-k^{\prime}}\left(t_{k}, t_{k^{\prime}}\right)$ as generating nonMarkovianity.

The approximation made in Eq. (13) is equivalent to having made a Trotter splitting on the full propagator in Eq. (8), prior to tracing over the baths. The error incurred by the approximation then depends upon the order of the splitting. In practice, we use a second-order splitting that incurs an error $\mathcal{O}\left(\Delta^{3}\right)$. We note that, since the superoperators in the products in Eq. (13) do not commute in general, the products seem ambiguous. In fact, demanding consistency of Eq. (13) with making a Trotter splitting in Eq. (8) fixes the order of multiplication of the superoperators to be from right to left with increasing $k$ and $k^{\prime}$. Whether the $k$ product or the $k^{\prime}$ product is performed first is irrelevant.

We now need a prescription for enforcing time ordering in Eq. (13). If each $\mathcal{I}_{k-k^{\prime}}\left(t_{k}, t_{k^{\prime}}\right)$ factorized into a product of time-local operators, this would be trivial, since we could just arrange the overall product of operators in order, e.g.,

$$
\overleftarrow{T} A\left(t_{k}\right) B\left(t_{k^{\prime}}\right)= \begin{cases}A\left(t_{k}\right) B\left(t_{k^{\prime}}\right) & \text { if } t_{k}>t_{k^{\prime}} \\ B\left(t_{k^{\prime}}\right) A\left(t_{k}\right) & \text { if } t_{k^{\prime}}>t_{k}\end{cases}
$$

To illustrate how we enforce time ordering generally, we now introduce some tensor network notation. A rank- $n$ tensor is represented as a node with $n$ legs and contractions are indicated by connecting legs. Operators are rank-2 tensors, so Eq. (15) has the tensor network representation

$$
\overleftarrow{T}\left[\begin{array}{ll}
j & j^{\prime} \\
\hline A & B \\
i & i^{\prime}
\end{array}\right]= \begin{cases}A & \text { if } t_{k}>t_{k^{\prime}} \\
A & \text { if } t_{k^{\prime}}>t_{k}\end{cases}
$$

with $i, j, i^{\prime}$, and $j^{\prime}$ labeling the components of the matrices $A\left(t_{k}\right)$ and $B\left(t_{k^{\prime}}\right)$, i.e., $\left[A\left(t_{k}\right)\right]_{i}^{j}$ and $\left[B\left(t_{k^{\prime}}\right)\right]_{i^{\prime}}^{j^{\prime}}$. On the left-hand side of Eq. (16) we can write the two disconnected rank-2 tensors as a single rank-4 (4-leg) tensor by using a tensor product, $C\left(t_{k}, t_{k^{\prime}}\right)=A\left(t_{k}\right) \otimes B\left(t_{k^{\prime}}\right)$. The tensor $C\left(t_{k}, t_{k^{\prime}}\right)$ would then be represented by a single node,

$$
\left[C\left(t_{k}, t_{k^{\prime}}\right)\right]_{i i^{\prime}}^{j j^{\prime}} \equiv{\frac{1}{i^{\mid}}}_{i^{\prime}}^{j}{ }^{j^{\prime}}
$$

with $\left[C\left(t_{k}, t_{k^{\prime}}\right)\right]_{i i^{\prime}}^{j j^{\prime}}=\left[A\left(t_{k}\right)\right]_{i}^{j}\left[B\left(t_{k^{\prime}}\right)\right]_{i^{\prime}}^{j^{\prime}}$. Comparing with Eq. (16), we see that enforcing time ordering on this tensor would correspond to contracting two of the legs together; either the legs labeled by $i$ and $j^{\prime}$ or those labeled by $j$ and $i^{\prime}$.

We can formalize the use of a tensor product to combine time-local operators by way of a history Hilbert space [47,48]. If $A\left(t_{k}\right)$ and $B\left(t_{k^{\prime}}\right)$ both act on a Hilbert space $\mathcal{H}$ spanned by a basis $\left\{\left|e_{i}\right\rangle\right\}$ then we can define a history space, $\mathcal{H}_{t_{k}} \otimes \mathcal{H}_{t_{k^{\prime}}}$, spanned by $\left\{\left|e_{i}\right\rangle \otimes\left|e_{i^{\prime}}\right\rangle\right\}$ on which their tensor product acts. Each subspace of the history space is identical to $\mathcal{H}$ and their corresponding bases are each identical to $\left\{\left|e_{i}\right\rangle\right\}$. In order to see how to write operators that depend on multiple time arguments in the history Hilbert space, let us consider an operator-valued function of the time-local operators $A\left(t_{k}\right)$ and $B\left(t_{k^{\prime}}\right)$, which we call $f\left[A\left(t_{k}\right) B\left(t_{k^{\prime}}\right)\right]$. This object exists in the familiar Hilbert space $\mathcal{H}$, and 
would have the usual matrix (i.e., rank-2 tensor) representation. It can be converted into an operator that acts on the history space by simply changing the regular product into a tensor product as follows:

$$
\begin{aligned}
D\left(t_{k}, t_{k^{\prime}}\right) & =f\left[A\left(t_{k}\right) \otimes B\left(t_{k^{\prime}}\right)\right] \\
& =\sum_{i i^{\prime} j j^{\prime}} D_{i i^{\prime}}^{i j^{\prime}}\left|e_{i}\right\rangle\left\langle e_{j}|\otimes| e_{i^{\prime}}\right\rangle\left\langle e_{j^{\prime}}\right|,
\end{aligned}
$$

with

$$
D_{i i^{\prime}}^{i j^{\prime}}=\left(\left\langlee_{i}\left|\otimes\left\langle e_{i^{\prime}}\right|\right) D\left(t_{k}, t_{k^{\prime}}\right)\left(\left|e_{j}\right\rangle \otimes\left|e_{j^{\prime}}\right\rangle\right)\right.\right.
$$

The operator $D\left(t_{k}, t_{k^{\prime}}\right)$ is a rank-4 tensor that is represented in the same way as $C\left(t_{k}, t_{k^{\prime}}\right)$ in Eq. (17). Crucially, to enforce time ordering on $D\left(t_{k}, t_{k^{\prime}}\right)$, i.e., to evaluate $\overleftarrow{T} f\left[A\left(t_{k}\right) B\left(t_{k^{\prime}}\right)\right]$, we simply connect together two of its legs, exactly as we did for $C\left(t_{k}, t_{k^{\prime}}\right)$.

The expression we wish to time order in Eq. (13) is a function of superoperators acting at $N+1$ different points in time. Thus, we construct the history space, $\mathfrak{L}_{H}$, as a product of $N+1$ copies of the Liouville space, $\mathfrak{L}$, in which the vectorized system density matrix, $\left.\left|\rho_{S}\right\rangle\right\rangle$, is defined. If the full discretized influence functional is converted to a superoperator on $\mathfrak{L}_{H}$, it is represented diagrammatically as a rank-2 $(N+1)$ tensor, the influence tensor, where each of the time points gives rise to a pair of legs. Time ordering is then enforced by connecting pairs of legs arising from consecutive time points, leaving two unconnected that are the two legs of the resulting dynamical map that is a rank2 tensor. So if we let $N=3$ then Eq. (13) has the tensor network representation

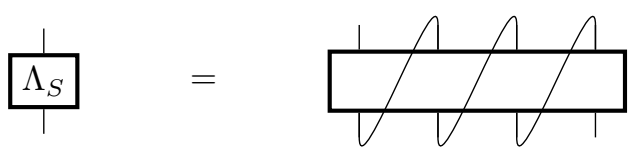

where each pair of legs on opposing sides of the influence tensor are those arising from a single subspace of $\mathfrak{L}_{H}$ and we have arranged the legs so that the rightmost pair correspond to $\mathfrak{L}_{t_{0}}$, the next pair along correspond to $\mathfrak{L}_{t_{1}}$, and so on.

Rather than representing Eq. (13) as a contraction of a single, large tensor in $\mathfrak{L}_{H}$, we note that each individual $\mathcal{I}_{k-k^{\prime}}\left(t_{k}, t_{k^{\prime}}\right)$ can be represented either as a rank-4 tensor in the subspace $\mathfrak{L}_{t_{k}} \otimes \mathfrak{L}_{t_{k^{\prime}}}\left(\right.$ for $\left.k \neq k^{\prime}\right)$ or as a rank-2 tensor in the subspace $\mathfrak{L}_{t_{k}}$ (for $k=k^{\prime}$ ). This means that the rank-2 $(N+1)$ influence tensor can be decomposed into a network of rank-2 and rank-4 tensors. For example, the string of tensor contractions corresponding to $N=3$ and a fixed $k^{\prime}=0$ in Eq. (13) would be represented as

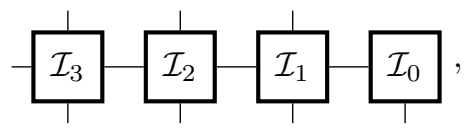

where contractions occur between the legs that act commonly on $\mathfrak{L}_{t_{0}}$. The full influence tensor in Eq. (13) with $N=3$, prior to time ordering, would then be written as

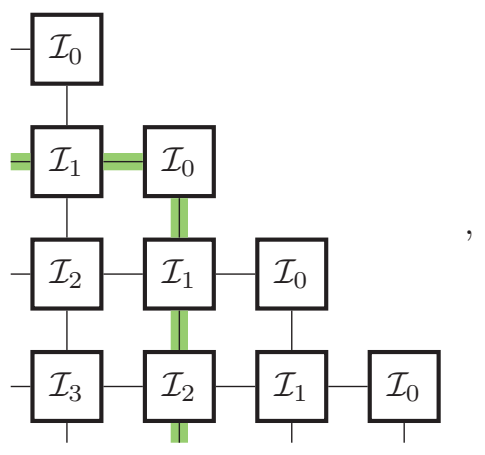

where we have highlighted in green a single string of contractions occurring through the $\mathfrak{L}_{t_{2}}$ subspace of $\mathfrak{L}_{H}$. The dangling legs along the bottom of (22) are the same as those on the bottom of the influence tensor in Eq. (20), while the legs along the left of (22) going from bottom to top are the same as those on the top in Eq. (20) going from right to left. The dimension of the bonds and the dangling legs in this network is $d^{2}$.

In the case where $\left[s_{\alpha}, s_{\alpha^{\prime}}\right]=0$ for all $\alpha, \alpha^{\prime}$ the nodes in (22) can be expanded in terms of the complete, shared eigenbasis of the system operators $s_{\alpha}$. Upon enforcing time ordering it is then possible to recover the standard path sum representation of the influence functional [44] and the network can be contracted efficiently using the original TEMPO algorithm [40], or by other means [45]. In the general case that the $s_{\alpha}$ do not commute, enforcing time ordering upon and contracting (22) efficiently has so far not been possible. We present a method that achieves this in the following section.

It should be pointed out that the influence tensor we have derived above is in fact a process tensor [41,45,49]. A process tensor is a mapping from the space of possible inputs to a system at a discrete set of times, for example a measurement or a preparation, to output states. Therefore, not only can (22) be used to calculate the dynamical map for the system but also all of its correlation functions $[45,50]$. Recently, it has been shown that this further allows the calculation of all correlation functions of the baths as well [51]. Thus, the tensor representation for the influence functional introduced here provides a way to completely characterize the full system-baths dynamics. 


\section{Matrix product form of the influence tensor}

Here we show how the influence tensor derived in the previous section can be efficiently put into the form of a matrix product operator (MPO). The first step is to convert each row of tensors in (22) individually into a MPO. This can be done via singular value decompositions (SVD) using the following routine:

(a)

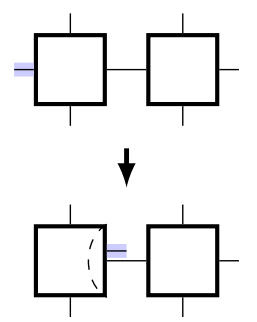

(b)

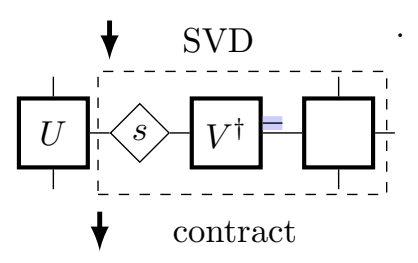

(d)

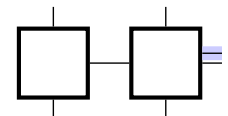

Here, we start with a pair of rank-4 tensors connected by a single leg as shown in (a). The desired outcome is shown in (d) with the dimension corresponding to the blue highlighted leg being stored by the right-hand tensor. This corresponds to an alternate decomposition of the rank- 6 tensor computed from contracting the bond in (a). The steps in routine (23) show how to switch to this representation without constructing the full tensor. In (b) we indicate with a dashed line that the legs of the tensor are partitioned into two groups; these are combined to form a matrix. We then perform a SVD on this matrix to arrive at step (c). Moving from (c) to (d) we contract the subnetwork indicated by the dashed box and in doing so arrive at the desired representation.

We can now use routine (23) to move the legs on the left of each row in (22), which puts each row in MPO form. For the bottom row, this looks like

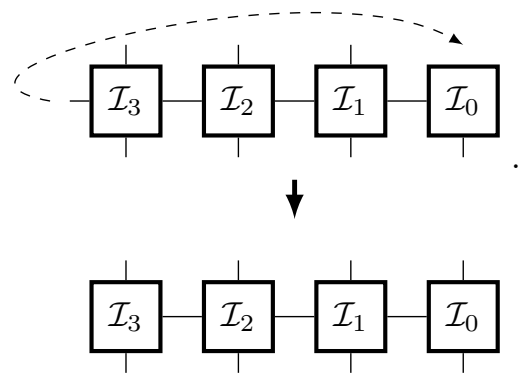

Having done this for each row, the full influence tensor can now be computed by iteratively contracting together the product of resulting MPOs, using SVDs to maintain an efficient representation:

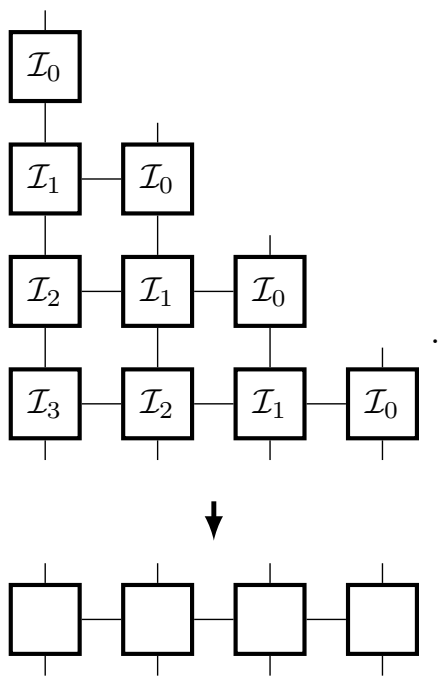

The result is a MPO representation of the influence tensor, where the vertical legs on each node correspond to the input (below) and output (above) legs of a single subspace of the full history space. That is, the rightmost node takes a state at $t_{0}$ as an input from below and outputs a state at $t_{1}$. The next node to the left would take this state from $t_{1}$ to $t_{2}$ and so on. Enforcing time ordering on the influence tensor to obtain the dynamical map, as in Eq. (20), is now straightforward. Doing this and acting the dynamical map on an initial state to obtain a final state at time $t_{4}$ would look like

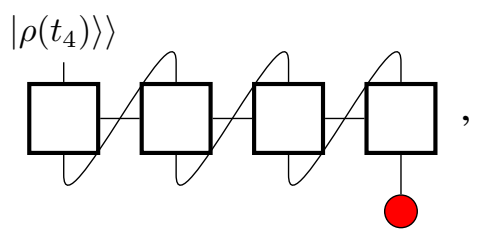

where the (rank-1) initial state is represented as a red circle.

There is one further step we can take to make the calculation more efficient. Instead of combining the individual influence functionals for each bath and then discretizing the results, as in Eq. (13), we can discretize each $\mathcal{F}_{\alpha}\left[s_{\alpha}^{L}, s_{\alpha}^{R}\right]$ in Eq. (10) separately, and obtain a MPO influence tensor for each of them individually using the procedure outlined above. The single MPO used to find the dynamical map in (26) is then calculated by contracting together the MPOs for each $\alpha$. In the case that there are just two baths, $\alpha_{1}$ and $\alpha_{2}$, the influence tensor MPO used in (26) would be 
written:

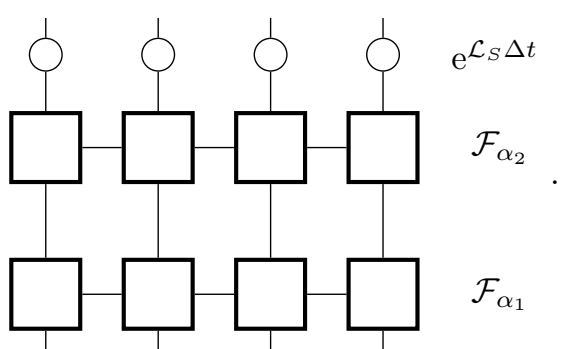

Here we have returned from the system interaction picture to the Schrödinger picture. The result of this is that the nodes in the bulk of the network are now defined in terms of time-independent system superoperators, e.g., $s_{\alpha}^{L}(t)=s_{\alpha}^{L}$, and free system propagators, rank-2 tensors represented as white circles, are applied in each subspace of the history space. The appearance of the free system propagators can be understood using the language of process tensors: in between the periods of time where the system interacts with the baths (the process) the operations we perform on the system are simply those of free evolution under the system Liouvillian. It is straightforward in this picture to see that a time-dependent system Liouvillian can be easily handled. This is done by simply discretizing the time dependence to steps of $\Delta t$ [50], which is already a parameter that is checked in convergence tests.

Calculating the influence tensor MPOs for each bath individually and then combining them does not result in a final MPO with lower bond dimension than if we had first combined the bath influence functionals and then directly calculated the full multibath MPO. This is to be expected since each method of calculation results in the same final MPO, up to the errors associated with Trotterization and SVD truncation. However, we find that the individual MPO calculations for each bath are computationally less intensive than the alternative, so we can do multiple easier calculations rather than a single intensive one.

The method we have introduced here can be taken even further by breaking down the MPOs for each bath into a series of MPOs representing the individual modes that make up the bath. This approach is similar in spirit to other recent works [34,52] where the process tensor can be constructed in a purely numerical manner, even when the environment is not Gaussian and the influence functional cannot be calculated analytically in closed form. Finally, we stress that process tensors calculated using different approaches for each environment can be combined like this, provided time is discretized in the same way. If the system coupling operators for different environments are related by a unitary transformation then the resulting process tensors can be transformed in the same way.

\section{Computing all dynamical maps efficiently}

The methodology we have presented so far enables us to calculate the dynamical map between two times, $t_{0}$ and $t_{N}$. In this section we explain how to extract dynamical maps between $t_{0}$ and all times $t<t_{N}$ during a single computation and give details on how we maximize the efficiency of the method. Generally, we aim to minimize the size of the tensors that must be stored while computing the dynamical maps. There are two steps we take toward this: we enforce time ordering on-the-fly during construction of the full multibath influence tensor and we keep contributions from each single-bath influence functional separate until absolutely necessary. We found this second point particularly helpful since combining influence tensors for noncommuting environments leads to a significant increase in bond dimension. The influence tensor is really a tensor representation of the integrand of a path sum that contains all possible trajectories the system takes while interacting with its environment. Contracting together two single-bath influence tensors combines two sets of such trajectories. If the couplings to these baths commute then many of these trajectories will be degenerate and the bond dimension remains low; otherwise, a larger bond dimension will be needed to store the combination of trajectories.

To recover a dynamical map from time $t_{0}$ to some time $t_{n}$ from an influence tensor constructed with a final time $t_{N}>t_{n}$, we need to discard from the tensor all correlations between pairs of time points that straddle the time $t_{n}$. We do this using a (normalized) vectorized identity matrix, $\left.d^{-1 / 2}|\mathbf{1}\rangle\right\rangle$, a rank-1 tensor that we call a trace cap since $\langle\langle\mathbf{1} \mid O\rangle\rangle=\operatorname{Tr}[O]$ for any vectorized system operator $O$. The trace cap is a null vector of any commutator superoperator, e.g., $\left.s_{\alpha}^{-}|\mathbf{1}\rangle\right\rangle=0$, and upon inspection of the form of Eq. (11), which is the exponent of an influence functional, we can see that the result of applying the trace caps to the influence tensor at time $t_{k}$ is to erase any temporal correlation that is generated between this time and all earlier times $t<t_{k}$. In practice, we use the trace cap as follows:

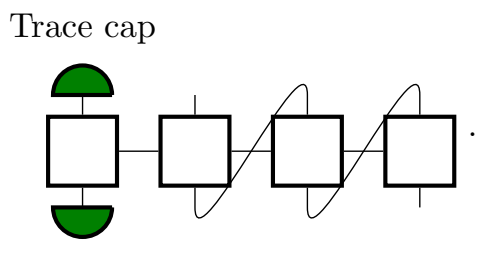

Here the trace caps are shown as green semicircles and we have extracted the dynamical map up to time $t_{3}$ from an influence tensor with $N=3$. Trace caps can be used in this way either on the full multibath influence tensor, as we did above, or on the single-bath influence tensors. For instance, if we write the influence tensor in (28) explicitly as a combination of two single-bath influence tensors and free system propagators, the trace caps are applied as 
follows:

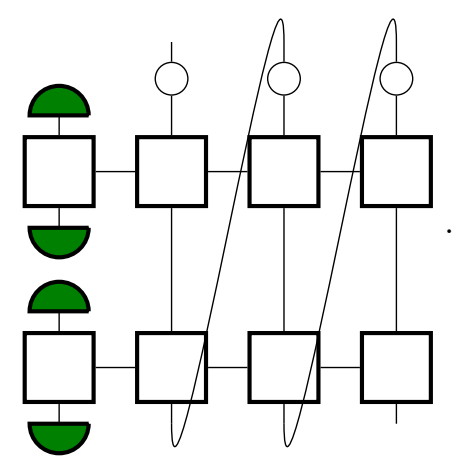

To see how we enforce time ordering on-the-fly, note that all the information required to calculate a dynamical map to time $t_{n}<t_{N}$ is contained in the first $n$ rows from the bottom in the influence tensor in the first diagram of (25). So, if we want to calculate the dynamical map from $t_{0}$ to $t_{2}$, we first need to contract together the first two rows, enforce time ordering only between the $t_{0}$ and $t_{1}$ nodes,

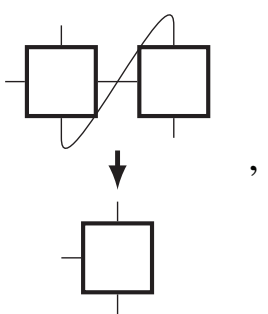

then apply trace caps to the rest of the MPO. Before applying the trace caps we store a copy of the MPO so that it can be used for further computation, but the time-ordering step here is permanent, since the legs that were contracted in this step, (30), have no further use in the calculation. Thus, the MPO we use to continue the calculation has only $N$ nodes, one fewer than the initial MPO we began with. Continuing to obtain the dynamical map to $t_{3}$, we contract our stored MPO with the third row in (25), use (30), then apply trace caps. After this we have stored a MPO that has $N-1$ nodes. In this way the MPO we store gets smaller as we proceed with the calculation.

Similarly to how we enforce time ordering as described above, we also combine single-bath influence tensors onthe-fly. To calculate the dynamical map from $t_{0}$ to $t_{n}$, we need to contract $n$ rows of (25) for each single-bath influence tensor, then we only need to combine the $n$ nodes of the resulting MPOs corresponding to times $t \leq t_{n-1}$ before applying time ordering on these nodes. After storing the remaining nodes of these separate MPOs, those for $t>t_{n-1}$, trace caps are used as in (29) to obtain the dynamical map. In practice, we did not contract the initial state with the influence tensor while calculating the dynamical maps. This allowed us to optimize our choice of initial state in order to find the steady state in as few timesteps as possible, which, since we considered two baths in our calculations, meant we also had to store a rank-4 tensor during computations. In summary, the following schematic tensor network shows which tensors we have stored after calculating the first $k$ dynamical maps:

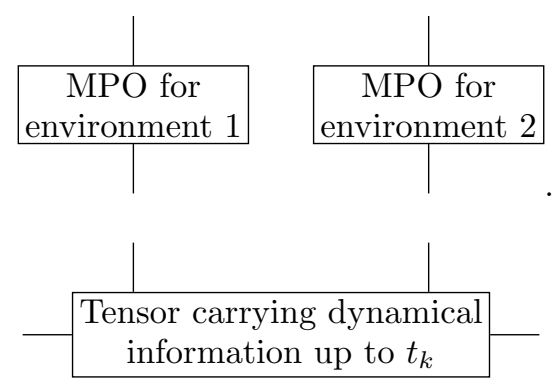

Each MPO is that computed by contracting the lower $k$ rows in Eq. (25). The dynamical map to time $t_{k}$ is found by contracting these MPOs into the rank- 4 tensor and then contracting trace caps into all of their remaining legs.

\section{E. Partial coarse graining}

There are three convergence parameters associated with each process tensor: the discretization timestep $\Delta$, the singular value threshold $\chi$ for truncation, and the memory length $K$ that dictates how many timesteps of system history are kept to capture the non-Markovian influence. In practice, this final approximation corresponds to setting $\mathcal{I}_{k>K}=\mathbb{1} \otimes \mathbb{1}$. For the two baths considered in this paper, the timescales of the different bath correlation functions are 2 orders of magnitude apart. Naïvely, the timestep appears to be fixed by the fastest bath (the optical bath in this case) and on this timescale there is no possibility of making a memory cutoff for the slower (vibrational) bath.

Considering how the separate process tensors are contracted, it might be thought that a larger timestep could be used for the slower bath with the same time-ordering contractions applied, i.e., with the faster bath being internally contracted, as in (30), several times between contractions with the slower bath. We find that, while this approach converges to the correct result, the error grows too quickly with increasing disparity in timesteps to be useful. We do, however, find a solution that enables us to propagate to much later times. Comparing Figs. 2(a) and 2(b) we illustrate how doubling the timestep manifests in both the discretization of Eq. (13) and the resulting tensor network. Figure 2(c) shows how we can discretize the inner and outer integrals of Eq. (13) with different timesteps. We refer to the timesteps of the inner and outer integrals as the history $\Delta_{h}$ and propagation $\Delta_{p}$ timesteps, respectively. For certain parameter regimes, we find that we achieve convergence with factors $\Delta_{h} / \Delta_{p}$ of the order of 100 , which allowed propagation lengths of the order of 1000 with no memory cutoff for the vibrational environment. We note however that the resources required by this algorithm still 
(a)

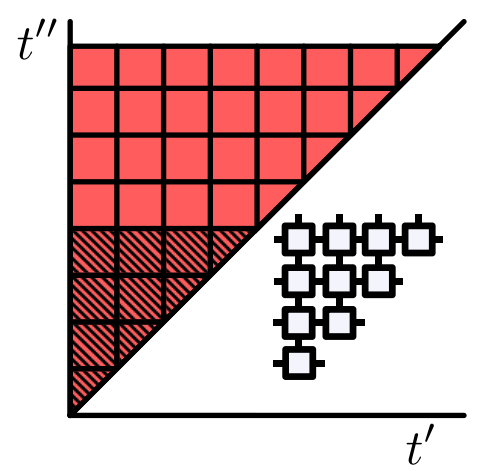

(b)

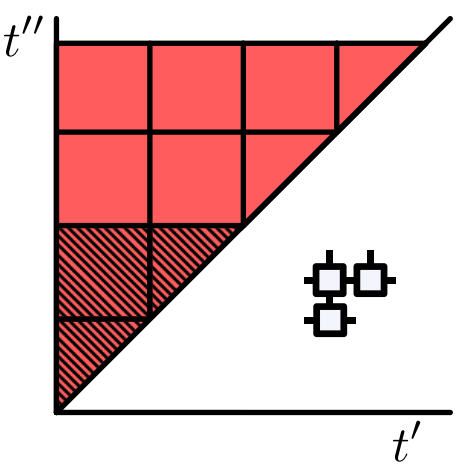

(c)

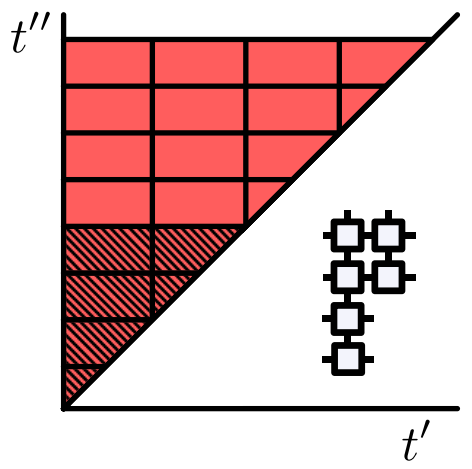

FIG. 2. Visualization showing the discretization of the double integral in Eq. (10) along with networks corresponding to the hatched areas. We have (a) the same timestep for both integrals, (b) same as (a) but with a timestep twice as large, and (c) the inner integral with a timestep twice that of the outer integral.

scale poorly with the system Hilbert space dimension and number of environments.

\section{INCOHERENT POPULATION TRANSFER}

\section{A. Hamiltonian}

As an example application of multibath TEMPO, we consider a single two-level dipole that is coupled to both an optical and vibrational environment. It has recently been shown that the two baths in this system are nonadditive, and when treated rigorously, can lead to population inversion of the two-level dipole [4]. Therefore, this is the ideal proving ground for testing our approach.

The Hamiltonian describing this system can be partitioned into system $(S)$, vibrational $(V)$, and optical $(O)$ parts as $H=H_{S}+H_{V}+H_{O}$. The system part,

$$
H_{S}=\delta \sigma^{+} \sigma^{-},
$$

describes the electronic states of the dipole with transition energy $\delta$. The vibrational part has two contributions:

$$
H_{V}=\sum_{\mathbf{k}} \omega_{\mathbf{k}} b_{\mathbf{k}}^{\dagger} b_{\mathbf{k}}+\sigma^{+} \sigma^{-} \sum_{\mathbf{k}}\left(g_{\mathbf{k}} b_{\mathbf{k}}^{\dagger}+g_{\mathbf{k}}^{*} b_{\mathbf{k}}\right) .
$$

Here the first term describes the energy of the bath alone and the second is the electron-phonon interaction term. The vibrational bath is composed of phonons of wavevector $\mathbf{k}$ and energy $\omega_{\mathbf{k}}$, with ladder operators $b_{\mathbf{k}}$ and $b_{\mathbf{k}}^{\dagger}$. Each of these modes couples to the dipole with strength $g_{\mathbf{k}}$ as described by the interaction term. The effect of the bath on the system is fully characterized by the phonon spectral density $J_{V}(\omega)=\sum_{\mathbf{k}}\left|g_{\mathbf{k}}\right|^{2} \delta\left(\omega-\omega_{\mathbf{k}}\right)$, the exact functional form of which will be considered in later sections. This interaction induces a displacement of the excited electronic manifold of the dipole that leads to Franck-Condon physics, that is, the nontrivial overlap of the vibrational wavefunctions between the manifolds $[4,53]$.
Finally, within the electric dipole approximation, the optical contribution to the Hamiltonian is given by

$$
H_{O}=\sum_{\mathbf{q}} v_{\mathbf{q}} a_{\mathbf{q}}^{\dagger} a_{\mathbf{q}}+\sigma^{x} \sum_{\mathbf{q}}\left(f_{\mathbf{q}} a_{\mathbf{q}}^{\dagger}+f_{\mathbf{q}}^{*} a_{\mathbf{q}}\right)
$$

where we have introduced the photon ladder operators $a_{\mathbf{q}}$ and $a_{\mathbf{q}}^{\dagger}$, corresponding to photons with energy $v_{\mathbf{q}}$ and wavevector q. This interaction term describes absorption or emission of a photon along with creation or annihilation of an exciton in the dipole. The light-matter coupling strength $f_{\mathbf{q}}$ leads to the optical spectral density $J_{O}(v)=$ $\sum_{\mathbf{q}}\left|f_{\mathbf{q}}\right|^{2} \delta\left(v-v_{\mathbf{q}}\right)$. The form of the optical spectral density is dependent on the gauge chosen for the light-matter interaction [54,55]. As we will see, whether or not it is possible for this model to exhibit population inversion is determined by the form of $J_{O}$. In this paper we use

$$
J_{O}(v)=\alpha \frac{v^{p}}{v_{c}^{p-1}} e^{-v / v_{c}}
$$

where $\alpha>0$ is the light-matter coupling strength constant. We have also introduced a phenomenological cutoff $v_{c}$ that is required for the influence functional in TEMPO to converge, and is also physically justified for finite systems $[55,56]$. The ohmicity of the spectral density $p$ is determined by the gauge choice and takes a positive value. In this work, we consider the two values of $p$ corresponding to two gauges that are most common to nonrelativistic quantum electrodynamics, $p=1$ (the Coloumb gauge) and $p=3$ (the multipolar gauge) $[54,55]$.

\section{B. Population inversion mechanism}

To give an intuitive understanding of the population inversion mechanism and demonstrate its reliance on nonadditivity of the two environments, we first consider a simplified four-level model shown in Fig. 3. This model 


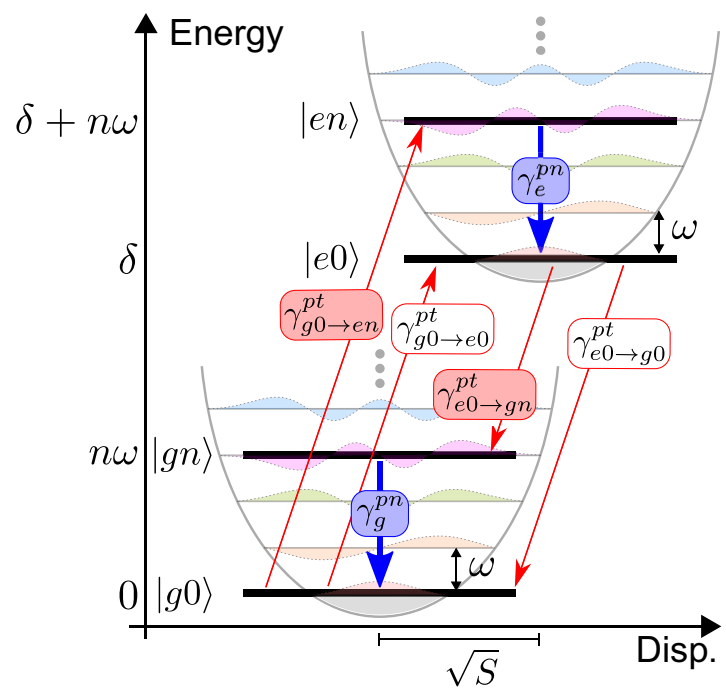

FIG. 3. The simple four-level model used to describe the mechanism of population inversion. Red and blue arrows denote photon and phonon transitions, respectively. The arrows with colored labels identify the pathway that creates population inversion. The symbols are defined in the main text.

consists of two electronic states separated by energy $\delta$, interacting with a single vibrational mode with frequency $\omega$ and coupling strength $g$. We then make three key approximations: we first assume that only two vibrational states contribute to the dynamics of the system, the zeroth and $n$th levels; second, the vibrational environment is assumed to be at zero temperature; and third, vibrational processes occur on a timescale much faster than optical transitions, such that photon emission and absorption only occur from the $|e 0\rangle$ and $|g 0\rangle$ states, as shown in Fig. 3.

Writing down a simple rate equation for the populations of the four-level system, and solving for the steady states, we find that the ratio of the steady-state populations of the electronic states is

$$
P=\frac{\gamma_{g 0 \rightarrow e 0}^{p t}+\gamma_{g 0 \rightarrow e n}^{p t}}{\gamma_{e 0 \rightarrow g 0}^{p t}+\gamma_{e 0 \rightarrow g n}^{p t}} .
$$

For population inversion, we require that $P>1$. Using Fermi's golden rule, we find the rates

$$
\begin{gathered}
\gamma_{g 0 \rightarrow e 0}^{p t}=2 \pi W_{0} J_{A}(\delta), \\
\gamma_{g 0 \rightarrow e n}^{p t}=2 \pi W_{n} J_{A}(\delta+n \omega), \\
\gamma_{e 0 \rightarrow g 0}^{p t}=2 \pi W_{0} J_{E}(\delta), \\
\gamma_{e 0 \rightarrow g n}^{p t}=2 \pi W_{n} J_{E}(\delta-n \omega),
\end{gathered}
$$

where $W_{j}=S^{j} \exp (-S) / j$ ! are the Franck-Condon factors accounting for the overlap of the displaced vibrational levels between manifolds differing in number by $j=0, n$, and $S=(|g| / \omega)^{2}$ is the dimensionless Huang-Rhys parameter. We have also introduced the emission and absorption optical spectral densities

$$
\begin{gathered}
J_{E}(v)=J_{O}(v)\left[1+N_{O}(v)\right], \\
J_{A}(v)=J_{O}(v) N_{O}(v),
\end{gathered}
$$

where $N_{O}(v)=\left[e^{v / T_{O}}-1\right]^{-1}$ is the population of the photon mode with energy $v$ at the temperature of the optical bath $T_{O}$.

When the vibrational coupling is weak $(S \approx 0)$, the manifolds are displaced only slightly such that $W_{0} \gg W_{n}$. In this limit

$$
\lim _{W_{0} \gg W_{n}} P=\frac{N_{O}(\delta)}{\left[1+N_{O}(\delta)\right]},
$$

where we have inserted Eq. (35) for $J_{O}$. This approaches 1 from below as $T_{O} \rightarrow \infty$ and so population inversion does not occur. Clearly then, strong vibrational coupling is key to population inversion and in this limit we instead find that

$$
\lim _{W_{n} \gg W_{0}} P \approx \frac{N_{O}(\delta+n \omega)(\delta+n \omega)^{p}}{\left[1+N_{O}(\delta-n \omega)\right](\delta-n \omega)^{p}} .
$$

Since $W_{n}$ is maximized for $n=S$ (here approximating $n$ as continuous), we should replace $n$ with $S$ in Eq. (40). From Eq. (40), we then see that the reason we need strong vibrational coupling is first to suppress optical transitions $\propto W_{0}$, i.e., to enhance the transition pathway identified by colored labels in Fig. 3, and second to increase the asymmetry in the transition energy of the decay and excitation rates along this pathway. This asymmetry can be enhanced in two additional ways: increasing either $T_{O}$ or $p$ to make $J_{E}$ and $J_{A}$ more sensitive to energy changes. Since population inversion is most easily achieved at high optical temperatures when $N_{O} \gg 1$, we focus on this limit and find that

$$
\lim _{\substack{W_{n} \gg W_{0} \\ T_{O} \rightarrow \infty}} P \rightarrow\left(\frac{\delta+S \omega}{\delta-S \omega}\right)^{p-1} .
$$

Here we see one very important prediction of the model: no matter how strong the vibrational coupling, population inversion cannot occur for $p \leq 1$. Since this is true at infinite optical temperature when inversion is easiest, this must be true for all temperatures. This result will be emphasized when we compare multibath TEMPO to the 
reaction coordinate and polaron calculations, introduced in the next section, across all parameter regimes in Sec. V.

Equation (41) is independent of the optical coupling strength. There are two reasons for this. First, the Fermi golden rule rates are only valid for small optical coupling. Second, we have assumed that the vibrational rates are significantly faster than the optical rates. In the next section, we derive the master equations for the polaron and RC techniques. The polaron technique similarly treats the optical coupling to second order and assumes that the vibrational bath is displaced instantaneously. This is the same as assuming that vibrational rates are large enough to lead to effectively instantaneous transitions. The RC technique relies on the weak light-matter coupling, but does not assume that the vibrational bath displaces instantaneously, and in Sec. V we find that population inversion decreases as optical lifetime becomes comparable to the displacement timescale.

\section{APPROXIMATE TECHNIQUES}

In this section we give a brief introduction to the two approximate techniques, a master equation that exploits the polaron transformation and the $\mathrm{RC}$ mapping. Both approaches are capable of capturing nonperturbative electron-phonon effects; however, they operate in two distinct regimes, as will be discussed below.

\section{A. Polaron theory}

Polaron theory accounts for strong electron-phonon interactions through a unitary transformation to the global Hamiltonian, such that $H_{P}=U H U^{\dagger}$, where $U=$ $\exp \left[-G \sigma^{+} \sigma^{-}\right]$with

$$
G=\sum_{\mathbf{k}}\left[\frac{g_{\mathbf{k}}}{\omega_{\mathbf{k}}} b_{\mathbf{k}}^{\dagger}-\frac{g_{\mathbf{k}}^{*}}{\omega_{\mathbf{k}}} b_{\mathbf{k}}\right] .
$$

This transformation dresses the excitonic degrees of freedom with vibrational modes of the environment forming a polaron, and providing a transformed basis in which to do perturbation theory. This has the advantage of providing a simple analytic expression for the master equation, while still accounting for strong system-environment interactions [17,57].

The resulting Hamiltonian in the polaron frame reads

$$
\begin{aligned}
H_{P}= & \delta_{P} \sigma^{+} \sigma^{-}+\sum_{\mathbf{k}} \omega_{\mathbf{k}} b_{\mathbf{k}}^{\dagger} b_{\mathbf{k}}+\sum_{\mathbf{q}} v_{\mathbf{q}} a_{\mathbf{q}}^{\dagger} a_{\mathbf{q}} \\
& +\left(B^{\dagger} \sigma^{+}+B \sigma^{-}\right) \sum_{\mathbf{q}}\left(f_{\mathbf{q}} a_{\mathbf{q}}^{\dagger}+f_{\mathbf{q}}^{*} a_{\mathbf{q}}\right),
\end{aligned}
$$

where the transformation has removed the linear vibrational coupling. The dipole transition operators are now dressed with the displacement operators $B=\exp [-G]$, such that $B^{\dagger} \sigma^{+}\left(B \sigma^{-}\right)$creates (destroys) a polaron with energy $\delta_{P}=\delta-\lambda$, where $\lambda=\int_{0}^{\infty} d \omega J_{V}(\omega) / \omega$ is the reorganization energy. The mathematical details of this transformation are well documented and can, for example, be found in Refs. [17-23,53,57-59].

To describe the dynamics generated from Eq. (43), we derive a Born-Markov master equation in which the optical and vibrational degrees of freedom are captured to second order in the polaron frame. In this particular example, the populations are independent of the coherences of the system, leading to a simple master equation of the form

$$
\dot{\rho}_{e e}(t)=\gamma_{\uparrow} \rho_{g g}(t)-\gamma_{\downarrow} \rho_{e e}(t),
$$

where $\rho_{e e}$ and $\rho_{g g}$ are the excited and ground-state populations in the lab frame. We have also defined the excitation $\gamma_{\uparrow}=\gamma\left(-\delta_{P}\right)$ and decay $\gamma_{\downarrow}=\gamma\left(\delta_{P}\right)$ rates in terms of the polaron rate function

$$
\gamma(\zeta)=2 \operatorname{Re} \int_{0}^{\infty} d t e^{i \zeta t} \mathcal{B}(t) \mathcal{C}(t)
$$

where the phonon correlation function is

$$
\mathcal{B}(t)=\operatorname{Tr}_{V}\left[B^{\dagger}(t) B(0) \rho_{V}\right]=\mathrm{e}^{-\phi(0)} \mathrm{e}^{\phi(t)},
$$

defined in terms of the Gibbs state of the vibrational environment, $\rho_{V}$, and the displacement operator in the interaction picture, $B(t)$. The phonon correlation function is characterized by the phonon propagator

$\phi(t)=\int_{0}^{\infty} d \omega \frac{J_{V}(\omega)}{\omega^{2}}\left[\operatorname{coth}\left(\frac{\omega}{2 T_{V}}\right) \cos (\omega t)-i \sin (\omega t)\right]$,

where $T_{V}$ is the temperature of the vibrational bath and we have taken a continuum limit for the phonon modes [17-19,21-23,53,57-59]. When comparing TEMPO with polaron theory, we take the spectral density

$$
J_{V}(\omega)=S \frac{\omega^{3}}{\omega_{c}^{2}} e^{-\omega / \omega_{c}},
$$

where $S$ is the dimensionless Huang-Rhys parameter and $\omega_{c}$ is the phonon cutoff frequency.

The optical contribution to the rate is given by the correlation function

$$
\begin{aligned}
\mathcal{C}(t) & =\sum_{\mathbf{q} \mathbf{q}^{\prime}} \operatorname{Tr}_{O}\left[A_{\mathbf{q}}^{\dagger}(t) A_{\mathbf{q}^{\prime}}(0) \rho_{O}\right] \\
& =\sum_{\mathbf{q}}\left|f_{\mathbf{q}}\right|^{2}\left\{N_{O}\left(v_{\mathbf{q}}\right) \mathrm{e}^{i \mathbf{v} t}+\left[N_{O}\left(v_{\mathbf{q}}\right)+1\right] \mathrm{e}^{-i v \mathbf{q}^{t}}\right\},
\end{aligned}
$$

where $\rho_{O}$ is the Gibbs state of the optical environment and $A_{\mathbf{q}}(t)$ is the operator $f_{\mathbf{q}} a_{\mathbf{q}}^{\dagger}+f_{\mathbf{q}}{ }^{*} a_{\mathbf{q}}$ in the interaction picture. Taking the continuum limit over the optical modes, 
we obtain an expression in terms of the absorption and emission effective spectral densities,

$$
\gamma(\zeta)=2 \int_{0}^{\infty} d v\left[J_{E}(v) \mathcal{V}(\zeta-v)+J_{A}(v) \mathcal{V}(\zeta+v)\right]
$$

where the vibrational influence is contained within [60-62]

$$
\mathcal{V}(\epsilon)=\mathrm{e}^{-\phi(0)} \operatorname{Re} \int_{0}^{\infty} d t \mathrm{e}^{\phi(t)} \mathrm{e}^{i \epsilon t} .
$$

The nonadditive nature of the vibrational and optical interactions is now clear because Eq. (50) is a convolution of optical functions with a vibrational function. Equation (50) can be solved numerically for the optical and vibrational spectral densities considered in this paper.

\section{B. Reaction coordinate}

An alternative semianalytic approach for describing the model outlined in Sec. III A is the RC method. Here key environmental degrees of freedom are included in an enlarged system Hamiltonian by way of a normal mode mapping [33,63-66]. The remaining environmental degrees of freedom are then captured through a residual environment, which can be treated to second order using a Born-Markov master equation $[32,66]$. This treatment allows one to access strong coupling and highly nonMarkovian regimes with the conceptual simplicity of a master equation, and little computational cost [67]. In this section we give an outline of the RC mapping and the subsequent derivation of the master equation. For more details, we refer the reader to Ref. [66].

We start by restricting the phonon environment to the case of an underdamped spectral density, of the form

$$
J_{V}(\omega)=\frac{\alpha_{\mathrm{UD}} \Gamma \omega_{0}^{2} \omega}{\left(\omega_{0}^{2}-\omega^{2}\right)^{2}+\Gamma^{2} \omega^{2}},
$$

which is one of the spectral densities for which the RC mapping is exact [32]. Here $\alpha_{\mathrm{UD}}$ is the reorganization energy, $\omega_{0}$ is the frequency of the spectral density's peak, and $\Gamma$ is its width.

Using a collective coordinate mapping, the vibrational contribution to the Hamiltonian given in Eq. (33) becomes $H_{V} \mapsto H_{\mathrm{RC}}+H_{\text {Res }}$, where

$$
\begin{gathered}
H_{\mathrm{RC}}=\eta \sigma^{+} \sigma^{-}\left(a^{\dagger}+a\right)+\Omega a^{\dagger} a \\
H_{\mathrm{Res}}=\left(a^{\dagger}+a\right) \sum_{\mathbf{k}} h_{\mathbf{k}}\left(c_{\mathbf{k}}^{\dagger}+c_{\mathbf{k}}\right)+\sum_{\mathbf{k}} \nu_{\mathbf{k}} c_{\mathbf{k}}^{\dagger} c_{\mathbf{k}} \\
+\left(a^{\dagger}+a\right)^{2} \sum_{\mathbf{k}} \frac{h_{\mathbf{k}}^{2}}{v_{\mathbf{k}}}
\end{gathered}
$$

Here we have introduced the annihilation (creation) operators $a\left(a^{\dagger}\right)$ and $c_{\mathbf{k}}\left(c_{\mathbf{k}}^{\dagger}\right)$ for the RC and residual environment, respectively. The mapped parameters can be written in terms of the unmapped spectral density, where $\eta=\sqrt{\pi \alpha_{\mathrm{UD}} \omega_{0} / 2}, \Omega=\omega_{0}$, and the residual environment is described by the spectral density $J_{\text {Res }}(\omega)=$ $\sum_{\mathbf{k}}\left|h_{\mathbf{k}}\right|^{2} \delta\left(\omega-v_{\mathbf{k}}\right)=\Gamma \omega / 2 \pi \Omega$.

Since the collective coordinate mapping acts only on the modes of the vibrational environment, leaving the system and optical degrees of freedom unchanged, then in the mapped frame we have $H=\tilde{H}_{S}+H_{\text {Res }}+H_{O}$, where we have defined the augmented system Hamiltonian as $\tilde{H}_{S}=H_{S}+H_{\mathrm{RC}}$.

A key advantage of the RC method is that we can treat the augmented system nonperturbatively, while treating the residual environment to second order, thus effectively capturing non-Markovian effects. To do this, we first apply the single-mode polaron transformation to Hamiltonian $U=$ $\exp \left[-v^{-1} \eta \sigma^{+} \sigma^{-}\left(a^{\dagger}-a\right)\right]$. This transformation diagonalizes the augmented system Hamiltonian such that $H_{S}^{\prime}=$ $U \tilde{H}_{S} U^{\dagger}=\delta \sigma^{+} \sigma^{-}+\Omega a^{\dagger} a$. In this transformed frame, we obtain a master equation of the form $\partial_{t} \rho(t)=\mathcal{L}[\rho(t)]$, with the Liouvillian

$$
\mathcal{L}[\rho(t)]=-i\left[\tilde{H}_{S}^{\prime}, \rho(t)\right]+\mathcal{K}_{\operatorname{Res}}[\rho(t)]+\mathcal{K}_{O}[\rho(t)],
$$

where $\rho(t)$ is the density operator for the augmented system, and $\mathcal{K}_{\text {Res }}$ is a superoperator representing the action of the residual environment [66]:

$$
\mathcal{K}_{\operatorname{Res}}[\rho]=[\mathcal{A}, \rho(t) \zeta]+\left[\zeta^{\dagger} \rho(t), \mathcal{A}\right]
$$

Here $\mathcal{A}=a^{\dagger}+a-2(\eta / v) \sigma^{+} \sigma^{-}$, and we have introduced the rate operator

$$
\zeta=\frac{\pi}{2} \sum_{\lambda} J_{\mathrm{Res}}(\lambda)\left[\operatorname{coth}\left(\frac{\lambda}{2 T_{V}}\right)+1\right] \mathcal{A}(\lambda),
$$

where the sum runs over the eigensplittings of the augmented system Hamiltonian, such that $\lambda \in\{ \pm \Omega, 0\}$, with the corresponding transition operators $\mathcal{A}(0)=$ $-2(\eta / \nu) \sigma^{+} \sigma^{-}, \mathcal{A}(\Omega)=a$, and $\mathcal{A}(-\Omega)=a^{\dagger}$.

Crucially, unlike standard perturbative treatments, the $\mathrm{RC}$ is capable of accounting for nonadditive effects when multiple environments are present [4]. In the case of an optical environment, this is done by explicitly deriving the optical contribution to the master equation in the eigenbasis of the augmented system. This results in a dissipator of the form

$$
\mathcal{K}_{O}[\rho(t)]=-\left[\sigma^{x},\left(\chi_{\uparrow}+\chi_{\downarrow}\right) \rho(t)\right]+\text { H.c. },
$$

where we have defined the rate operators corresponding to absorption $\chi_{\uparrow}=\sum_{j k} \sigma_{j k}^{x} \gamma_{\mathrm{RC}}^{\uparrow}\left(\lambda_{j k}\right)\left|\psi_{j}\right\rangle\left\langle\psi_{k}\right|$ and emission 
$\chi_{\downarrow}=\sum_{j k} \sigma_{j k}^{x} \gamma_{\mathrm{RC}}^{\downarrow}\left(\lambda_{j k}\right)\left|\psi_{j}\right\rangle\left\langle\psi_{k}\right|$. The rates for these processes are given as

$$
\begin{gathered}
\gamma_{\mathrm{RC}}^{\uparrow}(\lambda)=\pi J_{A}(\lambda) \Theta(\lambda), \\
\gamma_{\mathrm{RC}}^{\downarrow}(\lambda)=\pi J_{E}(-\lambda) \Theta(-\lambda),
\end{gathered}
$$

where $\Theta(x)$ is the Heaviside step function. Here, the steady state is independent of the Lamb shifts, allowing them to be neglected in the calculations we show.

If we compare the optical transition rates calculated in the RC model [Eqs. (59) and (60)], and those found in polaron theory in Eq. (50), we see some striking similarities, with vibronic contributions entering directly into the expressions for the rates. However, a key difference between the $\mathrm{RC}$ and polaron approach is that the displacement of the phonon environment in polaron theory is static, which leads to a breakdown of the perturbative master equation when the system dynamics approach the correlation time of the phonon environment [17]. In contrast, no such approximation is made in RC theory, and the displacement of the RC may vary throughout the system evolution, such that the method is valid in regimes of fast system dynamics. This will be significant in regimes where the system light-matter coupling dominates over the vibrational interaction.

\section{NONADDITIVE PHYSICS IN ALL COUPLING REGIMES}

With the methods described above, we may now explore the effects of nonadditivity on the nonequilibrium steady state of the two-level emitter. To do this, we separate the analysis into three regimes of optical coupling strength: weak, moderate, and strong. Weak coupling is defined as the thermalization timescale being shorter than the optical lifetime, and moderate coupling the reverse of this. In the strong coupling regime, a second-order truncation of the optical coupling breaks down and we use multibath TEMPO to explore this novel regime of light-matter coupling. In each regime we compare the steady-state population behavior predicted by the analytic models against multibath TEMPO. Analyzing the steady state exposes the most striking consequences of the nonadditive effects arising from our model. Furthermore, it has the added advantage of circumventing the awkward problem of defining equivalent initial states for a fair comparison of the short time dynamics; however, of course the dynamics are also available in all three approaches.

We present our comparisons for variable temperature of the optical field in Fig. 4 and as a function of light-matter coupling strength in Fig. 5. For all calculations, we use the optical spectral density defined in Eq. (35). For comparison with the polaron theory [Figs. 4(a) and 5(a)], we use the superohmic vibrational spectral density given in Eq. (48) as detailed in Sec. A, while Eq. (52) is used for the RC comparisons in Figs. 4(b) and 5(b).

\section{A. Weak optical coupling}

For small light-matter coupling strength, we see excellent agreement between both approximate methods and TEMPO, as is apparent from Fig. 4. This is expected as both approximate theories can account for electron-phonon

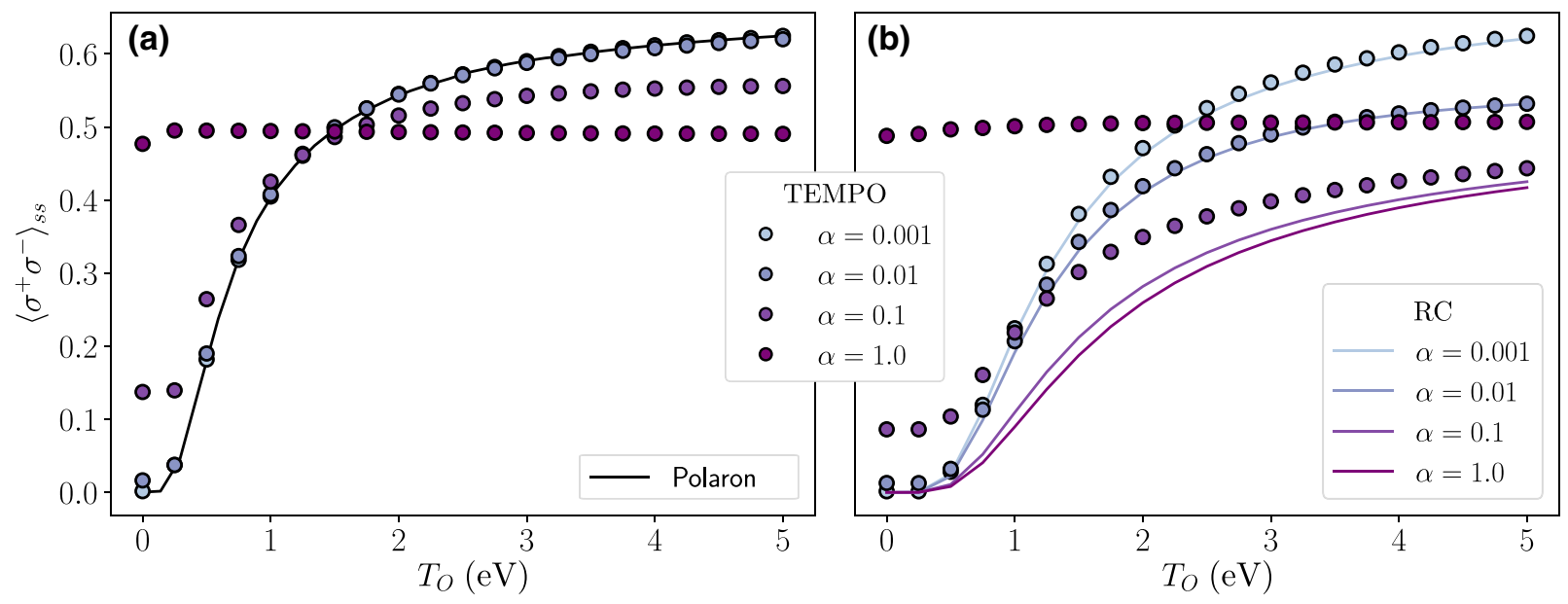

FIG. 4. Steady-state population as a function of the optical bath temperature $T_{O}$. In (a) we compare the populations as calculated by TEMPO and the polaron master equation for multiple optical couplings $\alpha$. The vibrational spectral density is of superohmic form with an exponential cutoff as given by Eq. (48). We fix the Huang-Rhys factor at $S=1$, the cutoff to $\omega_{c}=0.101 \mathrm{eV}$, and the polaron splitting to $\delta_{P}=1 \mathrm{eV}$. In (b) we plot the same comparison for the reaction coordinate (RC) master equation. The vibrational spectral density in this case is of Drude-Lorentz form as given by Eq. (52). We fix the coupling constant at $\alpha_{\mathrm{UD}}=0.3 \mathrm{eV}$, the linewidth at $\Gamma=0.1 \mathrm{eV}$, the mode frequency at $\omega_{0}=0.05 \mathrm{eV}$, and the polaron splitting at $\delta_{P}=2 \mathrm{eV}$. The other parameters for both (a) and (b) are $p=3, v_{c}=10 \mathrm{eV}$, and $T_{V}=0.0258 \mathrm{eV}$. 


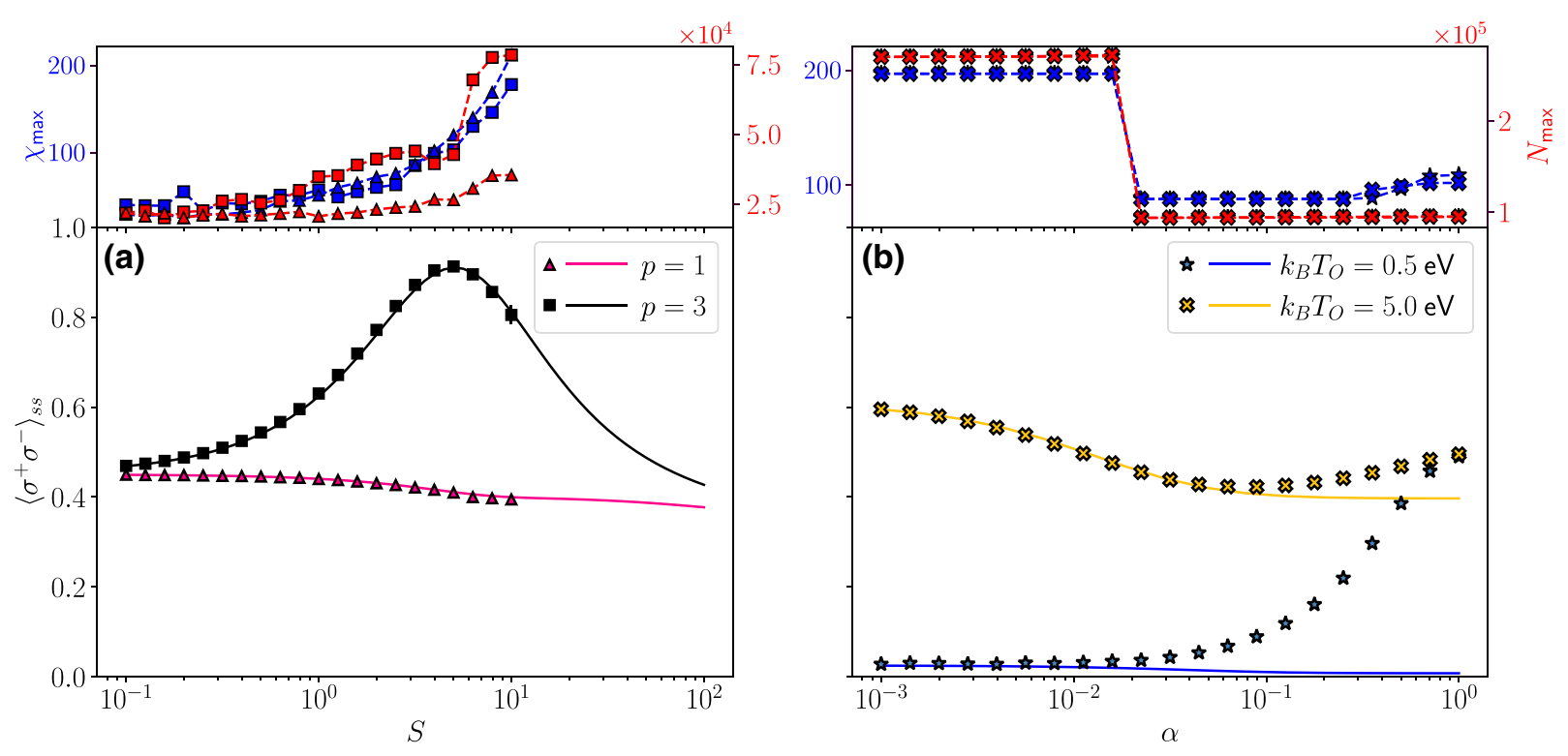

FIG. 5. Steady-state population as calculated using TEMPO (dots) and approximate techniques (lines). In (a) we compare TEMPO and the polaron master equation varying the Huang-Rhys factor for ohmicities $p=1$ and $p=3$ with fixed optical couplings, $\alpha=$ 0.0001 and $\alpha=0.001$, respectively, and temperature $T_{O}=5 \mathrm{eV}$. The other parameters for (a) are $\delta_{P}=1 \mathrm{eV}$ and $\omega_{c}=0.101 \mathrm{eV}$. In (b) we compare TEMPO and the RC master equation varying the optical coupling for two temperatures either side of the inversion transition. The other parameters for (b) are $\delta_{P}=2 \mathrm{eV}, p=3, \alpha_{\mathrm{UD}}=0.3 \mathrm{eV}, \Gamma=0.1 \mathrm{eV}$, and $\omega_{0}=0.05 \mathrm{eV}$. The common parameters for (a) and (b) are $v_{c}=10 \mathrm{eV}$ and $T_{V}=0.0258 \mathrm{eV}$. Above both (a) and (b) we also plot two measures of the size of the objects stored during the TEMPO runs [as illustrated in (31)]: the maximum bond dimension during the run, $\chi_{\max }$ (shown in blue), and the maximum number of elements contained in the tensors, $N_{\max }$ (shown in red). The markers correspond to the same parameters as in the main panels.

interactions beyond the second order, and the second-order treatment of the optical coupling is sufficient for small $\alpha$. Most striking from these figures is that, for small $\alpha$, all approaches predict a population inversion at large optical temperatures. This is as we expect from the work of Maguire et al. [4], as well as the four-level analysis in Sec. III B, where vibrational states induce an asymmetry in the optical rates, suppressing optical decay while enhancing the converse absorption process.

Using the polaron theory, we can also see that this behavior persists across a full range of vibrational couplings, agreeing well with TEMPO in Fig. 5(a). This is with the exception of the very largest couplings where there were difficulties with getting converged results from TEMPO. As well as the inherent difficulties associated with simulating stronger couplings, there is also a significant drop in the population transfer rates, meaning that longer propagation times are necessary to reach steady state. This suppression with strong vibrational coupling occurs because the system tries to excite into high lying vibrational states, requiring high-energy photon modes to be thermally populated to complete the transition. We have included error bars that correspond to the change in our steady-state prediction if we end the propagation 40 timesteps earlier to give an idea of how much we would expect the prediction to vary if we were to propagate out further. Taking these errors into account, this suggests that polaron theory predicts accurate steady-state populations over the full range of Huang-Rhys parameters.

We may also address the important prediction from the four-level model in Sec. III B that population inversion is conditional on the ohmicity of the optical field. This is confirmed by polaron theory and TEMPO in Fig. 5(a), where we see that population inversion never occurs when $p=1$, but does for $p=3$. Here we also find that a smaller value of $\alpha$ is required for $p=1$ in order to match the weak-coupling result from polaron theory.

\section{B. Moderate optical coupling}

At moderate optical coupling, where the optical timescales approach those of the phonon processes, we begin to see the population inversion predicted by TEMPO reduce. We attribute this to a dynamical undressing of the optical transition, where an emission and absorption of a photon occurs faster than polarons can be created or destroyed during transitions between the ground and excited states.

This is most apparent when comparing the polaron and TEMPO calculations in Fig. 4(a), where the steady-state population predicted by polaron theory is independent of the light-matter coupling strength $\alpha$. This can be seen from 
the polaron frame master equation in Eq. (44), where the steady-state population is dependent only on the ratio of $\gamma_{\uparrow} / \gamma_{\downarrow}$, which has no $\alpha$ dependence. Physically, this is a consequence of the static state-dependent displacement [Eq. (42)] applied to the phonon environment, regardless of the system dynamics. This and the approximations used to derive the master equation in Eq. (44), mean that within this theory it is always the case that a polaron is formed during an optical transition, regardless of how quickly this transition occurs. This results in the steady-state population being completely insensitive to the light-matter coupling strength. This is a common issue for polarontype master equations, and may be overcome by modifying the displacement through a variational treatment $[24,25]$. However, this is not straightforward when the two baths are out of equilibrium with each other.

In contrast, since the RC is included nonperturbatively in the augmented system, its state may respond to the increased optical transition rate, such that in regimes that polaron formation is not energetically favorable the twolevel emitter remains undressed by phonon modes. This flexibility in the theory means that RC continues to be in good agreement with TEMPO for moderate optical coupling strengths $(\alpha=0.01)$, as seen in Fig. 4(b).

\section{Strong optical coupling}

For large light-matter coupling strengths, the secondorder treatment used to derive the interaction with the optical field in both approximate techniques breaks down. As can be seen in Fig. 5(b), this leads to significant deviation between the RC method and multibath TEMPO, where at large $\alpha$ TEMPO predicts a trend toward $\left\langle\sigma^{+} \sigma^{-}\right\rangle=0.5$ independent of optical temperature $T_{O}$.

In the strong coupling limit a system-environment interaction can be thought of as a continuous measurement process where the system is consistently projected onto the eigenbasis of the coupling operator; the ultrastrong coupling limit $(\alpha \rightarrow \infty)$ can therefore be described using the quantum Zeno effect (QZE) [68,69]. In this limit the total system plus environments evolve under an effective Hamiltonian given by

$$
H_{\mathrm{QZE}}=\sum_{i} P_{i} H P_{i}
$$

where $P_{i}$ is a projection onto the $i$ th eigenstate of the optical interaction. Explicitly, this is defined as

$$
P_{i}=\left|\phi_{i}\right\rangle\left\langle\phi_{i}\right|,
$$

where

$$
\sigma^{x} \sum_{\mathbf{q}}\left(f_{\mathbf{q}} a_{\mathbf{q}}^{\dagger}+f_{\mathbf{q}}^{*} a_{\mathbf{q}}\right)\left|\phi_{i}\right\rangle=\phi_{i}\left|\phi_{i}\right\rangle
$$

These eigenstates can be partitioned into system and bath components and written as

$$
\left\{\left|\phi_{i}\right\rangle\right\}=\left\{|+\rangle \otimes\left|x_{i}\right\rangle,|-\rangle \otimes\left|x_{i}\right\rangle\right\}
$$

where $\{|+\rangle,|-\rangle\}$ are the eigenstates of $\sigma^{x}$ and $\left|x_{i}\right\rangle$ the eigenstates of $\sum_{\mathbf{q}}\left(f_{\mathbf{q}} a_{\mathbf{q}}^{\dagger}+f_{\mathbf{q}}{ }^{*} a_{\mathbf{q}}\right)$.

However, given that none of the other parts of the Hamiltonian act on the optical degrees of freedom, we only need to consider how the system component of $P_{i}$ affects the system operators in the Hamiltonian. This projection takes $\sigma^{+} \sigma^{-} \mapsto \mathbb{I}$ and leaves $\sigma^{x}$ unchanged such that we are left with an independent boson model. The steady state of $H_{\text {QZE }}$ in this case corresponds to a mixture of the eigenstates of $\sigma^{x}$; any such mixture corresponds to a population of 0.5 .

We can also note that the Hamiltonian is symmetric under the unitary transformation that leads to

$$
\left\{\sigma^{x}, \sigma^{y}, a_{\mathbf{q}}\right\} \rightarrow\left\{-\sigma^{x},-\sigma^{y},-a_{\mathbf{q}}\right\} .
$$

We would expect the steady state to also obey this symmetry, meaning that the $\sigma_{x}$ eigenstates must be equally populated. We can then conclude that in the strong coupling limit we expect the system steady state to correspond to a completely mixed state, i.e., $(|+\rangle\langle+|+|-\rangle\langle-|) / 2$. We have confirmed this prediction for the rightmost points of Fig. 5(b) by checking the corresponding system density matrix.

\section{Resource requirements}

The demands of the algorithm are characterized using the maximum bond dimension, $\chi_{\max }$, and maximum number of elements, $N_{\max }$, within the objects defined in (31) encountered over an entire simulation run.

In the upper panels of Fig. 5 we show both of these quantities for the corresponding TEMPO data points plotted below. We find that both $\chi_{\max }$ and $N_{\max }$ depend more strongly on the vibrational coupling $S$ than the optical coupling $\alpha$, increasing roughly linearly with $S$. We find that, generally, the resource requirements increase with the calculated relaxation time of the vibrationally dressed system. This is caused by the need to run the TEMPO algorithm out to much longer times than the relaxation time in order to achieve a converged steady state. This can be seen most dramatically looking at the variation of $\chi_{\max }$ and $N_{\max }$ with $\alpha$. At large $\alpha$ the system relaxes on a very short timescale, and as $\alpha$ is decreased, the system transition rate decreases, thus extending the relaxation time. We find that, for $\alpha \lesssim 10^{-2}$, we need to increase the propagation time, and then need a lower singular value threshold to achieve converged results, leading to the discontinuity in $\chi_{\max }$ and $N_{\text {max }}$. We choose the convergence parameters below the discontinuity to enable convergence of all lower values of 
$\alpha$, and hence there is little further variation. The singular value threshold in this region can be understood by noting that, as $\alpha \rightarrow 0$, the Hamiltonian becomes a pure dephasing model in the $\sigma_{z}$ basis and we need a greater precision to probe the perturbations to this trivial limit.

The results shown in Fig. 5(b) come from a model with a vibrational spectral density having more structure than that of (a), resulting in a longer relaxation time. Hence, the results in (b) generally required more resources to produce [67].

To estimate the added complexity of including a second environment, we computed the same resource measures for $S=0$, for both $p=1$ and $p=3$. These are much smaller, of the order of $\chi_{\max } \sim 10$ and $N_{\max } \sim 10^{3}$. A similar measure is meaningless for the $\alpha=0$ case as this corresponds to the exactly solvable independent boson model that does not have a uniquely defined steady state.

\section{CONCLUSIONS}

We have developed a new, exact technique based on tensor networks for finding the dynamics of a system that is coupled to multiple noncommuting baths. Our method can be used to model a broad range of couplings between the system and multiple baths described by arbitrary spectral densities. This has allowed us to probe previously unexplored regimes of simultaneous strong electron-phonon and light-matter coupling, highlighting the importance of proper treatment of nonadditive effects in nonequilibrium open quantum systems. In particular, we illustrate this by considering the incoherent population inversion predicted by Maguire et al. [4], which emerges in regimes of strong electron-phonon coupling.

Using multibath TEMPO, and backed by the analytic polaron and reaction coordinate approaches, we have highlighted two regimes of light-matter coupling. The first is a regime of dynamical undressing, where the light-matter coupling is sufficiently large for optical emission and absorption processes to occur on the same timescales as phonon interactions, preventing the formation of polaronlike states. The second regime occurs when the dynamics are dominated by the light-matter interaction, which leads to the optical environment projecting the system into the so-called Zeno subspace, causing decoherence with respect to the basis of $\sigma^{x}$. This results in steady-state populations that are independent of the temperature of the optical field.

The novel behavior described above is just one example of the nonadditive physics that emerges in nonequilibrium open quantum systems. Multibath TEMPO provides a powerful and versatile methodology for studying such phenomena, allowing the simulation of systems that are difficult to explore with other techniques. Furthermore, the general formulation of TEMPO presented can be applied to fermionic environments, where non-Markovian and nonadditive behaviors are expected to play an important role in the dynamical and steady-state behaviors of the system of interest [3,5].

The research data supporting this publication can be found in Ref. [70].

\section{ACKNOWLEDGMENTS}

We would like to thank Moritz Cygorek, Jonathan Keeling, and Gerald Fux for insightful discussions. D.G. and D.M.R. acknowledge studentship funding from the EPSRC (Grant No. EP/L015110/1). B.W.L. and E.M.G. acknowledge support from the EPSRC (Grants No. EP/T014032/1 and No. EP/T01377X/1). A.N. acknowledges support from an EPSRC Fellowship (EP/N008154/1). J.I.-S. acknowledges support from the Royal Commission for the Exhibition of 1851. A.S. acknowledges support from the Australian Research Council Centre of Excellence for Engineered Quantum Systems (EQUS, CE170100009). This work used EPCC's Cirrus HPC Service [71].

[1] G. G. Giusteri, F. Recrosi, G. Schaller, and G. L. Celardo, Interplay of different environments in open quantum systems: Breakdown of the additive approximation, Phys. Rev. E 96, 012113 (2017).

[2] J. Kołodyński, J. B. Brask, M. Perarnau-Llobet, and B. Bylicka, Adding dynamical generators in quantum master equations, Phys. Rev. A 97, 062124 (2018).

[3] M. T. Mitchison and M. B. Plenio, Non-additive dissipation in open quantum networks out of equilibrium, New J. Phys. 20, 033005 (2018).

[4] H. Maguire, J. Iles-Smith, and A. Nazir, Environmental Non-additivity and Franck-Condon Physics in Non-equilibrium Quantum Systems, Phys. Rev. Lett. 123, 093601 (2019).

[5] C. McConnell and A. Nazir, Electron counting statistics for non-additive environments, J. Chem. Phys. 151, 054104 (2019).

[6] G. J. Hedley, A. Ruseckas, and I. D. Samuel, Light harvesting for organic photovoltaics, Chem. Rev. 117, 796 (2017).

[7] A. Adronov and J. M. Fréchet, Light-harvesting dendrimers, Chem. Commun. 18, 1701 (2000).

[8] C. Felip-León, S. Díaz-Oltra, F. Galindo, and J. F. Miravet, Chameleonic, light harvesting photonic gels based on orthogonal molecular fibrillization, Chem. Mater. 28, 7964 (2016).

[9] E. Burzurí, J. O. Island, R. Díaz-Torres, A. Fursina, A. González-Campo, O. Roubeau, S. J. Teat, N. AliagaAlcalde, E. Ruiz, and H. S. van der Zant, Sequential electron transport and vibrational excitations in an organic molecule coupled to few-layer graphene electrodes, ACS Nano 10, 2521 (2016).

[10] G. D. Scholes, G. R. Fleming, A. Olaya-Castro, and R. Van Grondelle, Lessons from nature about solar light harvesting, Nature Chem. 3, 763 (2011).

[11] J. O. Thomas, B. Limburg, J. K. Sowa, K. Willick, J. Baugh, G. A. D. Briggs, E. M. Gauger, H. L. Anderson, and J. 
A. Mol, Understanding resonant charge transport through weakly coupled single-molecule junctions, Nat. Commun. 10, 1 (2019).

[12] J. K. Sowa, N. Lambert, T. Seideman, and E. M. Gauger, Beyond marcus theory and the Landauer-Büttiker approach in molecular junctions. II. A self-consistent born approach, J. Chem. Phys. 152, 064103 (2020).

[13] C. McConnell and A. Nazir, Strong coupling in thermoelectric nanojunctions: a reaction coordinate framework, arXiv preprint arXiv:2106.14799 (2021).

[14] C. Ciuti and I. Carusotto, Input-output theory of cavities in the ultrastrong coupling regime: The case of timeindependent cavity parameters, Phys. Rev. A 74, 033811 (2006).

[15] J. Koch and F. von Oppen, Franck-Condon Blockade and Giant Fano Factors in Transport through Single Molecules, Phys. Rev. Lett. 94, 206804 (2005).

[16] J. Koch, F. von Oppen, and A. V. Andreev, Theory of the franck-condon blockade regime, Phys. Rev. B 74, 205438 (2006).

[17] D. P. McCutcheon and A. Nazir, Quantum dot rabi rotations beyond the weak exciton-phonon coupling regime, New J. Phys. 12, 113042 (2010).

[18] C. Roy and S. Hughes, Phonon-Dressed Mollow Triplet in the Regime of Cavity Quantum Electrodynamics: Excitation-Induced Dephasing and Nonperturbative Cavity Feeding Effects, Phys. Rev. Lett. 106, 247403 (2011).

[19] C. Roy and S. Hughes, Polaron master equation theory of the quantum-dot mollow triplet in a semiconductor cavityQED system, Phys. Rev. B 85, 115309 (2012).

[20] I. Wilson-Rae and A. Imamoğlu, Quantum dot cavity-QED in the presence of strong electron-phonon interactions, Phys. Rev. B 65, 235311 (2002).

[21] M. Qin, H. Shen, X. Zhao, and X. Yi, Effects of systembath coupling on a photosynthetic heat engine: A polaron master-equation approach, Phys. Rev. A 96, 012125 (2017).

[22] D. M. Rouse, E. Gauger, and B. W. Lovett, Optimal power generation using dark states in dimers strongly coupled to their environment, New J. Phys. 21, 063025 (2019).

[23] F. A. Pollock, D. P. McCutcheon, B. W. Lovett, E. M. Gauger, and A. Nazir, A multi-site variational master equation approach to dissipative energy transfer, New J. Phys. 15, 075018 (2013).

[24] D. P. S. McCutcheon, N. S. Dattani, E. M. Gauger, B. W. Lovett, and A. Nazir, A general approach to quantum dynamics using a variational master equation: Application to phonon-damped rabi rotations in quantum dots, Phys. Rev. B 84, 081305 (2011).

[25] M. Bundgaard-Nielsen, J. Mørk, and E. V. Denning, Non-markovian perturbation theories for phonon effects in strong-coupling cavity quantum electrodynamics, Phys. Rev. B 103, 235309 (2021).

[26] B. M. Garraway, Nonperturbative decay of an atomic system in a cavity, Phys. Rev. A 55, 2290 (1997).

[27] B. J. Dalton, S. M. Barnett, and B. M. Garraway, Theory of pseudomodes in quantum optical processes, Phys. Rev. A 64, 053813 (2001).

[28] L. Mazzola, S. Maniscalco, J. Piilo, K.-A. Suominen, and B. M. Garraway, Pseudomodes as an effective description of memory: Non-markovian dynamics of two-state systems in structured reservoirs, Phys. Rev. A 80, 012104 (2009).

[29] D. Tamascelli, A. Smirne, S. F. Huelga, and M. B. Plenio, Nonperturbative Treatment of non-Markovian Dynamics of Open Quantum Systems, Phys. Rev. Lett. 120, 030402 (2018).

[30] G. Pleasance, B. M. Garraway, and F. Petruccione, Generalized theory of pseudomodes for exact descriptions of nonmarkovian quantum processes, Phys. Rev. Res. 2, 043058 (2020).

[31] F. Mascherpa, A. Smirne, A. D. Somoza, P. FernándezAcebal, S. Donadi, D. Tamascelli, S. F. Huelga, and M. B. Plenio, Optimized auxiliary oscillators for the simulation of general open quantum systems, Phys. Rev. A 101, 052108 (2020).

[32] J. Iles-Smith, A. G. Dijkstra, N. Lambert, and A. Nazir, Energy transfer in structured and unstructured environments: Master equations beyond the born-markov approximations, J. Chem. Phys. 144, 044110 (2016).

[33] A. Garg, J. N. Onuchic, and V. Ambegaokar, Effect of friction on electron transfer in biomolecules, J. Chem. Phys. 83, 4491 (1985).

[34] M. Cygorek, M. Cosacchi, A. Vagov, V. M. Axt, B. W. Lovett, J. Keeling, and E. M. Gauger, Numerically-exact simulations of arbitrary open quantum systems using automated compression of environments, arXiv:2101.01653 [quant-ph] (2021).

[35] R. Orús, A practical introduction to tensor networks: Matrix product states and projected entangled pair states, Ann. Phys. 349, 117 (2014).

[36] R. Orús, Tensor networks for complex quantum systems, Nat. Rev. Phys. 1, 538 (2019).

[37] A. W. Chin, Á. Rivas, S. F. Huelga, and M. B. Plenio, Exact mapping between system-reservoir quantum models and semi-infinite discrete chains using orthogonal polynomials, J. Math. Phys. 51, 092109 (2010).

[38] J. Prior, A. W. Chin, S. F. Huelga, and M. B. Plenio, Efficient Simulation of Strong System-Environment Interactions, Phys. Rev. Lett. 105, 050404 (2010).

[39] D. Tamascelli, A. Smirne, J. Lim, S. F. Huelga, and M. B. Plenio, Efficient Simulation of Finite-Temperature Open Quantum Systems, Phys. Rev. Lett. 123, 090402 (2019).

[40] A. Strathearn, P. Kirton, D. Kilda, J. Keeling, and B. W. Lovett, Efficient non-markovian quantum dynamics using time-evolving matrix product operators, Nat. Commun. 9, 3322 (2018).

[41] F. A. Pollock, C. Rodríguez-Rosario, T. Frauenheim, M. Paternostro, and K. Modi, Non-Markovian quantum processes: Complete framework and efficient characterization, Phys. Rev. A 97, 012127 (2018).

[42] N. Makri and D. E. Makarov, Tensor propagator for iterative quantum time evolution of reduced density matrices. I. Theory, J. Chem. Phys. 102, 4600 (1995).

[43] N. Makri and D. E. Makarov, Tensor propagator for iterative quantum time evolution of reduced density matrices. II. Numerical methodology, J. Chem. Phys. 102, 4611 (1995).

[44] A. Strathearn, Modelling non-Markovian Quantum Systems Using Tensor Networks (Springer International Publishing, Cham, Switzerland, 2020), p. 56. 
[45] M. R. Jørgensen and F. A. Pollock, Exploiting the Causal Tensor Network Structure of Quantum Processes to Efficiently Simulate non-Markovian Path Integrals, Phys. Rev. Lett. 123, 240602 (2019).

[46] A similar derivation that we are describing here also holds for interactions of the form $H_{I} \sim s^{\dagger} a+s a^{\dagger}$ for both fermionic and bosonic environments. In both cases the only substantive difference is in the form of Eq. (11).

[47] J. Cotler, C.-M. Jian, X.-L. Qi, and F. Wilczek, Superdensity operators for spacetime quantum mechanics, J. High Energy Phys. 2018, 93 (2018).

[48] R. B. Griffiths, Consistent Quantum Theory (Cambridge UP, Cambridge, 2002).

[49] S. Milz, C. Spee, Z.-P. Xu, F. A. Pollock, K. Modi, and O. Gühne, Genuine multipartite entanglement in time, SciPost Phys. 10, 141 (2021).

[50] G. E. Fux, E. P. Butler, P. R. Eastham, B. W. Lovett, and J. Keeling, Efficient Exploration of Hamiltonian Parameter Space for Optimal Control of non-Markovian Open Quantum Systems, Phys. Rev. Lett. 126, 200401 (2021).

[51] D. Gribben, A. Strathearn, G. E. Fux, P. Kirton, and B. W. Lovett, Using the environment to understand non-Markovian open quantum systems, arXiv:2106.04212 [quant-ph] (2021).

[52] E. Ye and G. K.-L. Chan, Constructing tensor network influence functionals for general quantum dynamics, arXiv:2101.05466 [quant-ph] (2021).

[53] P. Kok and B. W. Lovett, Introduction to Optical Quantum Information Processing (Cambridge university press, Cambridge, 2010).

[54] A. Stokes, A. Kurcz, T. P. Spiller, and A. Beige, Extending the validity range of quantum optical master equations, Phys. Rev. A 85, 053805 (2012).

[55] A. Stokes and A. Nazir, A master equation for strongly interacting dipoles, New J. Phys. 20, 043022 (2018).

[56] P. Drummond, Unifying the $p \cdot A$ and $d \cdot E$ interactions in photodetector theory, Phys. Rev. A 35, 4253 (1987).

[57] A. Nazir and D. P. McCutcheon, Modelling exciton-phonon interactions in optically driven quantum dots, J. Phys.: Condens. Matter 28, 103002 (2016).

[58] T. Brandes, Coherent and collective quantum optical effects in mesoscopic systems, Phys. Rep. 408, 315 (2005).

[59] A. Nazir, Correlation-Dependent Coherent to Incoherent Transitions in Resonant Energy Transfer Dynamics, Phys. Rev. Lett. 103, 146404 (2009).
[60] D. P. S. McCutcheon and A. Nazir, Model of the Optical Emission of a Driven Semiconductor Quantum dot: Phonon-Enhanced Coherent Scattering and Off-Resonant Sideband Narrowing, Phys. Rev. Lett. 110, 217401 (2013).

[61] K. Roy-Choudhury and S. Hughes, Spontaneous emission from a quantum dot in a structured photonic reservoir: Phonon-mediated breakdown of fermi's golden rule, Optica 2, 434 (2015).

[62] K. Roy-Choudhury and S. Hughes, Quantum theory of the emission spectrum from quantum dots coupled to structured photonic reservoirs and acoustic phonons, Phys. Rev. B 92, 205406 (2015).

[63] M. Thoss, H. Wang, and W. H. Miller, Self-consistent hybrid approach for complex systems: Application to the spin-boson model with debye spectral density, J. Chem. Phys. 115, 2991 (2001).

[64] K. H. Hughes, C. D. Christ, and I. Burghardt, Effectivemode representation of non-markovian dynamics: A hierarchical approximation of the spectral density. II. Application to environment-induced nonadiabatic dynamics, J. Chem. Phys. 131, 124108 (2009).

[65] R. Martinazzo, B. Vacchini, K. H. Hughes, and I. Burghardt, Communication: Universal markovian reduction of Brownian particle dynamics, J. Chem. Phys. 134, 011101 (2011).

[66] J. Iles-Smith, N. Lambert, and A. Nazir, Environmental dynamics, correlations, and the emergence of noncanonical equilibrium states in open quantum systems, Phys. Rev. A 90, 032114 (2014).

[67] D. Gribben, A. Strathearn, J. Iles-Smith, D. Kilda, A. Nazir, B. W. Lovett, and P. Kirton, Exact quantum dynamics in structured environments, Phys. Rev. Res. 2, 013265 (2020).

[68] P. Facchi and S. Pascazio, Quantum zeno dynamics: Mathematical and physical aspects, J. Phys. A: Math. Theor. 41, 493001 (2008).

[69] J. D. Cresser and J. Anders, Weak and ultrastrong coupling limits of the quantum mean force Gibbs state, arXiv:2104.12606 [quant-ph] (2021).

[70] D. Gribben, D. M. Rouse, J. Iles-Smith, A. Strathearn, H. Maguire, P. Kirton, A. Nazir, E. M. Gauger, and B. W. Lovett, Data for: "Exact dynamics of nonadditive environments in non-markovian open quantum systems," https://doi.org/10.17630/9bcdf917-20cc-4f9b-9a0b-15a1df $6 \mathrm{e} 78 \mathrm{c} 5$ (2020).

[71] https://www.epcc.ed.ac.uk/cirrus. 